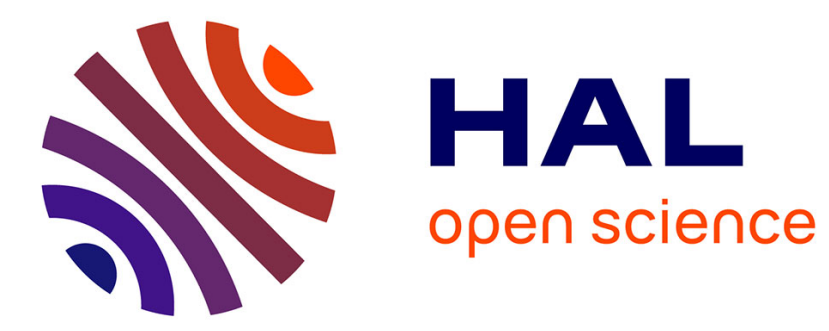

\title{
Uniqueness of vortexless Ginzburg-Landau type minimizers in two dimensions
}

\author{
Alberto Farina, Petru Mironescu
}

\section{To cite this version:}

Alberto Farina, Petru Mironescu. Uniqueness of vortexless Ginzburg-Landau type minimizers in two dimensions. Calc. Var. Partial Differential Equations, 2013, 46 (3-4), pp.523-554. hal-00747410

\author{
HAL Id: hal-00747410 \\ https://hal.science/hal-00747410
}

Submitted on 31 Oct 2012

HAL is a multi-disciplinary open access archive for the deposit and dissemination of scientific research documents, whether they are published or not. The documents may come from teaching and research institutions in France or abroad, or from public or private research centers.
L'archive ouverte pluridisciplinaire HAL, est destinée au dépôt et à la diffusion de documents scientifiques de niveau recherche, publiés ou non, émanant des établissements d'enseignement et de recherche français ou étrangers, des laboratoires publics ou privés. 


\title{
Uniqueness of vortexless Ginzburg-Landau type minimizers in two dimensions
}

\author{
Alberto Farina* $\quad$ Petru Mironescu ${ }^{\dagger}$
}

November 3, 2011

\begin{abstract}
In a simply connected two dimensional domain $\Omega$, we consider Ginzburg-Landau minimizers $u$ with zero degree Dirichlet boundary condition $g \in H^{1 / 2}\left(\partial \Omega ; \mathbb{S}^{1}\right)$. We prove uniqueness of $u$ whenever either the energy or the Ginzburg-Landau parameter are small. This generalizes a result of Ye and Zhou requiring smoothness of $g$. We also obtain uniqueness when $\Omega$ is multiply connected and the degrees of the vortexless minimizer $u$ are prescribed on the components of the boundary, generalizing a result of Golovaty and Berlyand for annular domains. The proofs rely on new global estimates connecting the variation of $|u|$ to the Ginzburg-Landau energy of $u$. These estimates replace the usual global pointwise estimates satisfied by $\nabla u$ when $g$ is smooth, and apply to fairly general potentials. In a related direction, we establish new uniqueness results for critical points of the Ginzburg-Landau energy.
\end{abstract}

\section{Introduction}

The aim of this paper is to generalize and unify several known existence results for minimizers or critical points of the simplified Ginzburg-Landau (GL, in short) energy

$$
E_{\varepsilon}(u)=\frac{1}{2} \int_{\Omega}|\nabla u|^{2}+\frac{1}{4 \varepsilon^{2}} \int_{\Omega}\left(1-|u|^{2}\right)^{2} .
$$

Here,
a) $\Omega \subset \mathbb{R}^{2}$ is a $C^{1}$ bounded domain.
b) $u: \Omega \rightarrow \mathbb{C}$.
c) $\varepsilon>0$ is a (not necessarily small) parameter.

We will consider two types of boundary conditions:

a) Dirichlet boundary condition: given $g: \partial \Omega \rightarrow \mathbb{S}^{1}$, we prescribe $u=g$ on $\partial \Omega$.

b) Degree boundary condition: let $\Gamma_{j}, j \in \llbracket 0, k \rrbracket$, be the components of $\partial \Omega$. Given the integers $d_{j}$, we prescribe $|u|=1$ on $\partial \Omega$ and $\operatorname{deg}\left(u, \Gamma_{j}\right)=d_{j}, j \in \llbracket 0, k \rrbracket$.

In the case of the Dirichlet boundary condition, uniqueness holds for $\varepsilon>\frac{1}{\sqrt{\lambda_{1}}}$, where $\lambda_{1}$ is the first eigenvalue of $-\Delta$ in $H_{0}^{1}(\Omega)$. Uniqueness need not hold for small $\varepsilon$ [4]. However, Ye and Zhou [26] proved the following

${ }^{*}$ LAMFA, CNRS UMR 6140, Université de Picardie Jules Verne, Faculté de Sciences, 33, rue Saint Leu, 80039 Amiens CEDEX 1, France. Email address: Alberto.Farina@u-picardie.fr $\bowtie$

†Université de Lyon; Université Lyon 1; INSA de Lyon, F-69621; Ecole Centrale de Lyon; CNRS, UMR5208, Institut Camille Jordan, 43 blvd du 11 novembre 1918, F-69622 Villeurbanne-Cedex, France. Email address: mironescu@math.univ-lyon1.fr 
1.1 Theorem ([26]). Assume that $\Omega$ is a smooth bounded simply connected domain and that $g$ is smooth and of zero degree.

a) For small $\varepsilon, E_{\varepsilon}$ has only one minimizer with Dirichlet boundary condition $g$.

b) If, in addition, $g$ is sufficiently close to a constant, ${ }^{1}$ then uniqueness holds for each $\varepsilon$.

The proof in [26] relies on global pointwise estimates on the gradients of minimizers. ${ }^{2}$ In particular, some smoothness is required on $g$. However, the natural space for $g$ is $H^{1 / 2}\left(\partial \Omega ; \mathbb{S}^{1}\right)$, and for such $g$ minimizers $u$ need not satisfy uniform gradient estimates up to the boundary. Our first result is the following

1.2 Theorem. Assume that $\Omega$ is $C^{1}$ and simply connected. Let $g \in H^{1 / 2}\left(\partial \Omega ; \mathbb{S}^{1}\right)$ have zero degree.

a) For small $\varepsilon, E_{\varepsilon}$ has only one minimizer with Dirichlet boundary condition $g$.

b) There is some explicit universal constant $\delta$ such that if $g$ has an $\mathbb{S}^{1}$-valued extension $v$ to $\Omega$ satisfying $\int_{\Omega}|\nabla v|^{2} \leq \delta$, then the minimizers of $E_{\varepsilon}$ with Dirichlet boundary condition $g$ are unique for each $\varepsilon$.

Item b) will be discussed in more detail in Section 8. For the moment, let us simply mention that the condition on $v$ is a smallness condition on the $H^{1 / 2}$ seminorm of $g$, and thus b) in Theorem 1.2 generalizes b) in Theorem 1.1.

1.3 Remark. In the above theorem, we may take $\delta=0.04518303544 \ldots$ The same holds for Theorem 1.5.

We next turn to the case of prescribed degrees. Before proceeding further, let us note that minimizers with prescribed degrees are never unique: if $u$ is a minimizer, then so it $\alpha u$, for each $\alpha \in \mathbb{S}^{1}$. Thus, at best, we can hope uniqueness modulo $\mathbb{S}^{1}$. Our starting point is the following result of Golovaty and Berlyand [14].

1.4 Theorem ([14]). Let $\Omega=\{z \in \mathbb{C} ; 1<|z|<R\}$. Let $d \geq 1$ be an integer. Then there is some $R_{d}>1$ such that, for $1<R<R_{d}$ and for each $\varepsilon, E_{\varepsilon}$ has, modulo $\mathbb{S}^{1}$, a unique minimizer with prescribed degrees $d$ and $d$ on the two circles of $\partial \Omega$.

Our result is the following.

1.5 Theorem. Let $\mathfrak{M}$ be the infimum of the energy $E_{\varepsilon}$ with given prescribed degrees. ${ }^{3}$ There is some explicit universal constant $\delta$ such that, if $\mathfrak{M}<\delta$, then the infimum is attained and the minimizer is unique (modulo $\mathbb{S}^{1}$ ).

We emphasize the fact that existence of minimizers of $E_{\varepsilon}$ is part of the statement; in general, $\mathfrak{M}$ is not attained [1, Example 1].

Our result is related to Theorem 1.4 as follows: consider, as a test function in the setting of Theorem 1.4, the map $z \mapsto \frac{z^{d}}{|z|^{d}}$. This gives $\mathfrak{M} \leq \pi d^{2} \ln R$. Thus $\mathfrak{M} \leq \delta$ for $R$ close to 1 , and Theorem 1.4 follows from Theorem 1.5.

The proof of Theorem 1.5 is completely different in nature from the one of Theorem 1.4 in [14]. In [14], the authors use in an essential way a geometric property of circular annuli, property which need not hold for arbitrary domains. Instead, we develop here an approach based on some new global estimates, estimates which do not see the geometry of the domain. The main tool in the proof of Theorems 1.2 and 1.5 is Theorem 3.6, which is of independent interest. Theorem 3.6 gives a lower bound for the energy required, for a GL minimizer $u$, to move from $|u| \equiv 1$ on the boundary to its minimum value in $\Omega$. Here is a striking special case of Theorem 3.6.

\footnotetext{
${ }^{1}$ The precise condition in [26] is $\int_{\partial \Omega}\left(\left|\frac{\partial g}{\partial \tau}\right|+\left|\frac{\partial^{2} g}{\partial \tau^{2}}\right|\right) \leq \delta$, where $\delta=\delta(\Omega)$ is sufficiently small.

${ }^{2}$ It is common knowledge in the GL community that $|u|$ close to 1 plus global pointwise estimates lead to uniqueness.

${ }^{3}$ Thus $\mathfrak{M}$ depends on $\Omega$, on $\varepsilon$ and on the degrees.
} 
1.6 Theorem. Let $\Omega$ be simply connected. Let $u$ minimize $E_{\varepsilon}$ with respect to its own boundary condition $g \in H^{1 / 2}\left(\partial \Omega ; \mathbb{S}^{1}\right)$. If $u$ vanishes somewhere in $\Omega$, then $\int_{\Omega}|\nabla u|^{2} \geq 2 \pi$.

The value $2 \pi$ is sharp.

We emphasize the fact that this is not a statement about $H^{1}$ maps. $H^{1}$ maps can move from 1 to 0 at almost zero cost. The above property is specific to GL minimizers.

Heuristics and outline of the proofs. Let us first consider, in a simply connected domain $\Omega$, the minimization problem

$$
\min \left\{\frac{1}{2} \int_{\Omega}|\nabla u|^{2} ; u: \Omega \rightarrow \mathbb{S}^{1}\right\}
$$

with, say, Dirichlet boundary condition $g: \partial \Omega \rightarrow \mathbb{S}^{1}$. This nonlinear problem is equivalent to a linear one. Indeed, each test function $u$ may be written as $u=e^{\imath \varphi}$, with $\operatorname{tr} \varphi$ given (in function of $g$ ) on $\partial \Omega$ [9], and the minimization problem becomes

$$
\min \left\{\frac{1}{2} \int_{\Omega}|\nabla \varphi|^{2} ; \varphi: \Omega \rightarrow \mathbb{R}\right\}
$$

with a Dirichlet boundary condition. Uniqueness of a $u$-minimizer follows now from the strict convexity of the $\varphi$-energy. ${ }^{4}$ In contrast, there is no similar trick transforming the GinzburgLandau energy $E_{\varepsilon}$ into a strictly convex one. However, it is possible to obtain a sort of logconvexity of $E_{\varepsilon}$ in a neighborhood of a minimizer of modulus close to 1 . When the boundary condition is smooth, this is hidden in the proof of Ye and Zhou [26] and explained in [22]. Our results cover the case of an arbitrary boundary datum, respectively the case of a multiply connected domain. The assumptions of the main results (Theorems 1.2 and 1.5) guarantee precisely the fact that minimizers have modulus close to $1 .^{5}$ In each of these results, the plan of the proof is

1. Prove that a minimizer $u_{\varepsilon}$ has modulus close to 1 .

2. Prove the following substitute to log-convexity: if $v_{\varepsilon}$ is another minimizer, then $E_{\varepsilon}\left(v_{\varepsilon}\right)>$ $E_{\varepsilon}\left(u_{\varepsilon}\right)$ unless $v_{\varepsilon}=u_{\varepsilon}{ }^{6}$

When the energy $E_{\varepsilon}\left(u_{\varepsilon}\right)$ is small, Step 1 . is obtained via Theorem 3.6, which basically asserts precisely that small energy GL minimizers have modulus close to 1 . When the energy is not supposed to be small, we complete Step 1. under the assumption that $\varepsilon$ is small. In this case we use a clearing out technique; this well-known approach $[4,23,17,18,5]$ is revisited in the special case we are interested in in Section 7.

Unlike the approach in [26], where Step 2. is obtained via pointwise estimates, ${ }^{7}$ our approach relies on global estimates. These estimates are either the Wente estimates [25] used in their sharp form derived by Bethuel and Ghidaglia [6] (used for small energy solutions), or asymptotic versions of the Wente estimates (used for arbitrary energy solutions); a typical such result is Lemma 4.3 .

The main auxiliary result, Theorem 3.6 , is proved according to the following lines. Consider a minimizer $u_{\varepsilon}$ of $E_{\varepsilon}$, and let $t=\min |u|$.

1. Assume first that the level line $[|u|=m]$, with $m>t$, is connected. Then we prove that this line is "not too short", for otherwise $|u|$ has no reason to reach the value $t$. This implies that the variation of $|u|$ on this level line is "not too small". Integrating over $m$ the lower bound of the energy on the level line $[|u|=m]$ and using the co-area formula, we obtain a lower bound for the energy.

2. In general, the level line $[|u|=m]$ has no reason to be connected. However, if this line is "not too small", then we still obtain the same conclusion as above.

\footnotetext{
${ }^{4}$ In other words, the initial $u$-energy is log-convex.

${ }^{5}$ In particular, our results do not apply to the case where minimizers do have zeros. In this case, uniqueness need not hold [4], and, even if it holds, the proof of uniqueness requires completely different arguments [20, 22].

${ }^{6}$ It is known to experts that a similar approach is useful in the proof of Krasnosel'skii's uniqueness result [15].

${ }^{7}$ Such estimates need not hold under the assumptions of Theorems 1.2 and 1.5.
} 
3. It remains to treat the case where this line is "small". In this case, we are able to fall back into Step 1. above and conclude again. The tool allowing this is an origami style result (the Contraction Lemma) asserting the existence of a convenient folding of the plane.

Our paper is organized as follows. In Section 2, we prove the Contraction Lemma (Lemma 2.1) crucial in the proof of Theorem 3.6. Section 3 is devoted to the proof of Theorem 3.6. In Section 4, we recall the sharp Wente estimates of Bethuel and Ghidaglia [6] and establish some new related estimates, which will be used in the proof of Theorem 1.2. Section 5 collects some standard results about lifting and degree. In Section 6, we prove Theorem 1.5. Clearing out results required in the proof of Theorem 1.2 are gathered in Section 7. The proof of Theorem 1.2 is presented in Section 8. Finally, in Section 9, we will discuss other uniqueness results, in the spirit of [12] or [1]. This relies on some additional clearing out results, established at the beginning of the section.

Acknowledgment. The authors thank the anonymous referee for his careful reading of the proof and useful suggestions.

\subsection{Notation, definitions}

a) $\mathbb{D}(z, r)$ is the open disc of center $z$ and radius $r$. $\mathbb{D}_{r}=\mathbb{D}(0, r)$, and $\mathbb{D}=\mathbb{D}_{1}$ is the unit disc.

b) $C(z, r)$ is the circle of center $z$ and radius $r$. $C_{r}=C(0, r)$, and $\mathbb{S}^{1}=C_{1}$.

c) $\ell(I)$ is the length of the (smooth) curve $I$.

d) A contraction is a 1-Lipschitz (i.e., non dilating) map.

e) If $A, B \in \mathbb{S}^{1}$ are such that $B \neq-A$, then $\overparen{A B}$ denotes the smallest arc of $\mathbb{S}^{1}$ with endpoints $A$ and $B$. If $B=-A$, then $\overparen{A B}$ is one of the two half circles with endpoints $A$ and $B$.

f) Let $s, t \geq 0$. We denote by $\mathscr{A}_{s, t}$ the area of $\left\{z \in \mathbb{D}_{t} ; \operatorname{Re} z>s\right\}$ and by $\ell_{s, t}$ the length of $\left\{z \in C_{t} ; \operatorname{Re} z>s\right\}$.

g) $\langle$,$\rangle denotes the scalar product in \mathbb{R}^{2}$. In particular, $\left\langle r e^{\imath \theta}, s e^{\imath \varphi}\right\rangle=r s \cos (\theta-\varphi)$.

h) If $a, b \in \mathbb{Z}$, then $\llbracket a, b \rrbracket=\{n \in \mathbb{Z} ; a \leq n \leq b\}$.

i) $\wedge$ denotes the vector product of vectors in $\mathbb{R}^{2}$ (or complex numbers): $\left(a_{1}, a_{2}\right) \wedge\left(b_{1}, b_{2}\right)=$ $a_{1} b_{2}-a_{2} b_{1}$. If $u=u_{1}+\imath u_{2}$ is a function of two-variables, then we set $u \wedge \nabla u=u_{1} \nabla u_{2}-$ $u_{2} \nabla u_{1}$.

j) Jac denotes the Jacobian determinant (in $\left.\mathbb{R}^{2}\right)$ : Jac $(g, h)=\nabla g \wedge \nabla h$.

k) The interior of a simple closed plane curve $\gamma$ is the domain enclosed by $\gamma$.

\section{Contents}

1 Introduction $\quad 1$

1.1 Notation, definitions . . . . . . . . . . . . . . . . 4

2 Contraction lemma $\quad 5$

3 Lower bounds for the energy of rotational minimizers $\quad 8$

4 Wente estimates and applications $\quad 12$

5 Degree, lifting, and the Ginzburg-Landau equation $\quad 14$

6 Proof of Theorem 1.5 18

7 Clearing out $\quad 19$

8 Proof of Theorem $1.2 \quad 22$ 
9 More $\quad 23$

9.1 GL critical points with Dirichlet boundary condition . . . . . . . . . . . 23

9.2 GL critical points with prescribed degrees . . . . . . . . . . . . . . 26

\section{Contraction lemma}

This section is devoted to the proof of the following

2.1 Lemma (Contraction lemma). Let $I_{1}, \ldots, I_{l}$ be arcs of $C_{\rho}$ such that $\sum \ell\left(I_{j}\right) \leq \pi \rho$. Then there is a map $\mathbb{P}: \mathbb{R}^{2} \rightarrow \mathbb{R}^{2}$ such that:

a) Restricted to each $I_{j}, \mathbb{P}$ acts like a rotation $\mathscr{R}_{j}$.

b) $\mathbb{P}$ is a contraction.

c) $\mathbb{P}\left(I_{1} \cup \ldots \cup I_{l}\right)$ is connected.

d) $|\mathbb{P}(z)|=|z|, \forall z \in \mathbb{R}^{2}$.

Proof. We may assume $\rho=1$. We may also assume that the $I_{j}$ 's are mutually disjoint.

It suffices to construct a map $\mathbb{V}: \mathbb{S}^{1} \rightarrow \mathbb{S}^{1}$ such that

a) Restricted to each $I_{j}, \mathbb{V}$ is a rotation.

b) $\mathbb{V}$ is a contraction.

c) $\mathbb{V}\left(I_{1} \cup \ldots \cup I_{l}\right)$ is connected.

Indeed, assume that such $\mathbb{V}$ exists. We may then take

$$
\mathbb{P}(z)=|z| \mathbb{V}(z /|z|), \forall z \in \mathbb{C} \backslash\{0\}, \mathbb{P}(0)=0,
$$

and conclude via the following

2.2 Lemma. Let $\mathbb{V}: \mathbb{S}^{1} \rightarrow \mathbb{S}^{1}$ be a contraction and let $\mathbb{P}$ be defined by (2.1). Then $\mathbb{P}$ is a contraction.

Proof of Lemma 2.2. Since $\mathbb{V}$ is a contraction, we have $|V(\omega)-\mathbb{V}(\eta)|^{2} \leq|\omega-\eta|^{2}$, and thus

$$
-\langle\mathbb{V}(\omega), \mathbb{V}(\eta)\rangle \leq-\langle\omega, \eta\rangle, \quad \forall \omega, \eta \in \mathbb{S}^{1} .
$$

If $r, s \geq 0$ and $\omega, \eta \in \mathbb{S}^{1}$, then

$$
|\mathbb{P}(r \omega)-\mathbb{P}(s \eta)|^{2}=r^{2}+s^{2}-2 r s\langle\mathbb{V}(\omega), \mathbb{V}(\eta)\rangle \leq r^{2}+s^{2}-2 r s\langle\omega, \eta\rangle=|r \omega-s \eta|^{2} .
$$

Proof of Lemma 2.1 continued. We establish the existence of $\mathbb{V}$ by induction on $l$, the case $l=1$ being obvious. Assuming the cases $1, \ldots, l-1$ settled, it suffices to construct some $\mathbb{T}: \mathbb{S}^{1} \rightarrow \mathbb{S}^{1}$ such that:

a) Restricted to each $I_{j}, \mathbb{T}$ is a rotation.

b) $\mathbb{T}$ is a contraction.

c) $\mathbb{T}\left(I_{1} \cup \ldots \cup I_{l}\right)$ has at most $l-1$ connected components.

Indeed, assume such $\mathbb{T}$ exists. Apply the induction hypothesis to the components of $\mathbb{T}\left(I_{1} \cup \ldots \cup\right.$ $I_{l}$ ), and let $\mathbb{Y}$ be the corresponding contraction. Then $\mathbb{V}=\mathbb{Y} \bigcirc \mathbb{T}$ has all the desired properties.

Before constructing $\mathbb{T}$, let us gather some elementary considerations, stated as Lemmata 2.3-2.6. The first one is straightforward.

2.3 Lemma. Let $A, B, A^{\prime}, B^{\prime} \in \mathbb{S}^{1}$. Then

$$
\left|A^{\prime}-B^{\prime}\right| \leq|A-B| \Longleftrightarrow \ell\left(\widehat{A^{\prime} B^{\prime}}\right) \leq \ell(\widehat{A B}) .
$$


2.4 Lemma. Write $\mathbb{S}^{1}=I \cup J$, where $I, J$ are closed arcs with disjoint interiors. Let $\mathbb{\mathbb { V }}: \mathbb{S}^{1} \rightarrow \mathbb{S}^{1}$. Then $\mathbb{T}$ is a contraction on $\mathbb{S}^{1}$ if and only if $\mathbb{T}$ is a contraction on I and on $J$, .

Proof of Lemma 2.4. It suffices to prove the if part. This amounts to the inequality

$$
|\mathbb{T}(A)-\mathbb{T}(B)| \leq|A-B| \quad \text { if } A \in I \text { and } B \in J .
$$

By continuity, we may further assume that $B \neq-A$. By (2.2), inequality (2.3) amounts to $\ell(\overline{\mathbb{T}(A) \mathbb{T}(B)}) \leq \ell(\widehat{A B})$. Let $C \in \partial I=\partial J$ be such that $\widehat{A B}=\widehat{A C} \cup \widehat{C B}$. Since $A, C \in I$ and $B, C \in J$, we find that

$$
\ell(\overline{\mathbb{T}(A) \mathbb{T}(B)}) \leq \ell(\overline{\mathbb{T}(A) \mathbb{T}(C)})+\ell(\overline{\mathbb{T}(C) \mathbb{T}(B)}) \leq \ell(\widehat{A C})+\ell(\widehat{C B})=\ell(\widehat{A B}) .
$$

2.5 Lemma. Let $\mathbb{B}: \mathbb{R} \rightarrow \mathbb{R}$ be a $2 \pi$-periodic contraction. Let $\mathbb{\mathbb { V }}: \mathbb{S}^{1} \rightarrow \mathbb{S}^{1}, \mathbb{T}\left(e^{\imath \theta}\right)=e^{\imath \mathbb{B}(\theta)}$. Then $\mathbb{T}$ is a contraction.

Proof of Lemma 2.5. Let $\omega, \eta \in \mathbb{S}^{1}$. We may write $\omega=e^{\imath \theta}, \eta=e^{\imath \varphi}$, with $|\theta-\varphi| \leq \pi$. Since $\mathbb{B}$ is a contraction, we have $\mathbb{B}(\theta)-\mathbb{B}(\varphi)=t(\theta-\varphi)$ for some $t \in[-1,1]$. We find that

$$
|\mathbb{T}(\omega)-\mathbb{T}(\eta)|^{2}=2-2 \cos (\mathbb{B}(\theta)-\mathbb{B}(\varphi))=2-2 \cos (t(\theta-\varphi)) \leq 2-2 \cos (\theta-\varphi)=|\omega-\eta|^{2} .
$$

2.6 Lemma. Let $L_{1}, \ldots, L_{l}$ be closed intervals on the real line, with disjoint interiors and such that their union is an interval, say $L=L_{1} \cup \ldots \cup L_{l}=[a, b]$. Set $\ell_{j}=\ell\left(L_{j}\right), j \in \llbracket 1, l \rrbracket$. Let $M \subset \llbracket 1, l \rrbracket$ be a non empty set of indices and let $\alpha, \beta \in \mathbb{R}$.

The following are equivalent:

a) There is a contraction $\mathbb{X}: L \rightarrow \mathbb{R}$ such that $\mathbb{X}(a)=\alpha, \mathbb{X}(b)=\beta$ and, restricted to each $L_{j}$ with $j \in M, \mathbb{X}$ is a translation.

b) It holds that

$$
\sum_{j \in M} \ell_{j}-\sum_{j \notin M} \ell_{j} \leq \beta-\alpha \leq \sum_{j \in M} \ell_{j}+\sum_{j \notin M} \ell_{j} .
$$

Proof of Lemma 2.6. a) $\Longrightarrow$ b) We may assume that $L_{j}=\left[x_{j}, x_{j+1}\right]$, with $x_{1}=a$ and $x_{l+1}=b$. Then

$$
\begin{aligned}
\beta-\alpha & =\mathbb{X}\left(x_{l+1}\right)-\mathbb{X}\left(x_{1}\right)=\sum\left(\mathbb{X}\left(x_{j+1}\right)-\mathbb{X}\left(x_{j}\right)\right) \\
& =\sum_{j \in M}\left(\mathbb{X}\left(x_{j+1}\right)-\mathbb{X}\left(x_{j}\right)\right)+\sum_{j \notin M}\left(\mathbb{X}\left(x_{j+1}\right)-\mathbb{X}\left(x_{j}\right)\right) \\
& =\sum_{j \in M} \ell_{j}+\sum_{j \notin M}\left(\mathbb{X}\left(x_{j+1}\right)-\mathbb{X}\left(x_{j}\right)\right) \in\left[\sum_{j \in M} \ell_{j}-\sum_{j \notin M} \ell_{j}, \sum_{j \in M} \ell_{j}+\sum_{j \notin M} \ell_{j}\right] .
\end{aligned}
$$

b) $\Longrightarrow$ a) Let $t \in[-1,1]$ be such that $\beta-\alpha=\sum_{j \in M} \ell_{j}+t \sum_{j \notin M} \ell_{j}$. Then the continuous piecewise linear map $\mathbb{X}$ satisfying $\mathbb{X}(a)=\alpha$ and having, in $L_{j}$, slope 1 if $j \in M$, respectively slope $t$ if $j \notin M$, has all the required properties.

Proof of Lemma 2.1 completed. We consider, on $\mathbb{S}^{1}$, the direct orientation with respect to $\mathbb{D}$. We may assume that, with respect to this orientation, the $\operatorname{arcs} I_{j}$ are ordered $I_{1}, \ldots, I_{l}$. Let $M_{j}$ be the arc of $\mathbb{S}^{1}$ placed after $I_{j}$ and before $I_{j+1}$ (with the convention $I_{l+1}=I_{1}$ ). Equivalently, the initial point of $M_{j}$ is the final point of $I_{j}$, and the final point of $M_{j}$ is the initial point of $I_{j+1}$. Let $\ell_{j}=\ell\left(I_{j}\right)$ and $\mathfrak{q}_{j}=\ell\left(M_{j}\right)$.

Our purpose is to construct, for a value of $k \in \llbracket 1, l \rrbracket$ to be determined later, a contraction $\mathbb{T}: \mathbb{S}^{1} \rightarrow \mathbb{S}^{1}$ such that:

a) $\mathbb{T}$ is the identity on $I_{k+1}$. 
b) $\mathbb{T}$ is a rotation on each $I_{j}$.

c) $\mathbb{T}\left(I_{k}\right)$ intersects $I_{k+1}$.

Indeed, assume that, for at least a value $k \in \llbracket 1, l \rrbracket$, such a $\mathbb{T}$ exists. Then $\mathbb{T}\left(I_{1} \cup \ldots \cup I_{l}\right)$ has at most $l-1$ connected components. As explained before the statement of Lemma 2.3, this allows us to complete the proof of Lemma 2.1.

Let us first derive a necessary and sufficient condition for the existence of such $\mathbb{T}$ when $k=1$; we next transpose this condition to the other values of $k$. The final argument will imply that this is possible for at least a value of $k$ in $\llbracket 1, l \rrbracket$.

We may assume that $I_{1}$ starts at $z=1$. In view of Lemmata 2.5, 2.6, and of the fact that the exponential map changes translations into rotations, existence of $\mathbb{T}$ is equivalent to the existence of a contraction $\mathbb{B}:[0,2 \pi] \rightarrow \mathbb{R}$ such that:

a) $\mathbb{B}(0)=\mathbb{B}(2 \pi)$.

b) $\mathbb{B}$ is the identity on $\left\{\ell_{1}+\mathfrak{q}_{1}\right\}+\left[0, \ell_{2}\right]$.

c) $\mathbb{B}$ is of the form $\theta \mapsto \theta+a_{j}$ on each segment $\left\{\sum_{n<j}\left(\ell_{n}+\mathfrak{q}_{n}\right)\right\}+\left[0, \ell_{j+1}\right]$.

d) $\mathbb{B}\left(\left[0, \ell_{1}\right]\right)$ intersects $\left\{\ell_{1}+\mathfrak{q}_{1}\right\}+\left[0, \ell_{2}\right]$.

Let $x=\mathbb{B}(0)=\mathbb{B}(2 \pi)$. In view of Lemma 2.6 , existence of $\mathbb{B}$ satisfying a)-c) on $\left[0, \ell_{1}+\ell_{2}+\mathfrak{q}_{1}\right]$ is equivalent to

$$
\ell_{1}+\ell_{2}-\mathfrak{q}_{1} \leq \ell_{1}+\ell_{2}+\mathfrak{q}_{1}-x \leq \ell_{1}+\ell_{2}+\mathfrak{q}_{1}
$$

i.e., to

$$
0 \leq x \leq 2 \mathfrak{q}_{1} .
$$

On the other hand, condition d) is equivalent to

$$
\mathfrak{q}_{1} \leq x \leq \ell_{1}+\ell_{2}+\mathfrak{q}_{1} .
$$

Finally, existence of $\mathbb{B}$ satisfying a)-c) on $\left[\ell_{1}+\ell_{2}+\mathfrak{q}_{1}, 2 \pi\right]$ together with $\mathbb{B}(2 \pi)=x$ amounts to

$$
\sum_{j \neq 1,2} \ell_{j}-\sum_{j \neq 1} \mathfrak{q}_{j} \leq x-\left(\ell_{1}+\ell_{2}+\mathfrak{q}_{1}\right) \leq \sum_{j \neq 1,2} \ell_{j}+\sum_{j \neq 1} \mathfrak{q}_{j},
$$

which is equivalent to

$$
\sum \ell_{j}-\sum \mathfrak{q}_{j}+2 \mathfrak{q}_{1} \leq x \leq 2 \pi .
$$

In view of inequalities (2.5)-(2.7), existence of $\mathbb{B}$ for some $k$ is equivalent to

$$
\max \left\{0, \mathfrak{q}_{k}, \sum \ell_{j}-\sum \mathfrak{q}_{j}+2 \mathfrak{q}_{k}\right\} \leq \min \left\{2 \mathfrak{q}_{k}, \ell_{k}+\ell_{k+1}+\mathfrak{q}_{k}, 2 \pi\right\}
$$

or, equivalently, to

$$
\max \left\{\mathfrak{q}_{k}, \sum \ell_{j}-\sum \mathfrak{q}_{j}+2 \mathfrak{q}_{k}\right\} \leq \min \left\{2 \mathfrak{q}_{k}, \ell_{k}+\ell_{k+1}+\mathfrak{q}_{k}\right\}
$$

Inequality (2.8) amounts to four inequalities. It is easy to see that two of them are automatically satisfied, and that the two others are

$$
\sum \ell_{j}-\sum \mathfrak{q}_{j}+2 \mathfrak{q}_{k} \leq 2 \mathfrak{q}_{k},
$$

respectively

$$
\sum \ell_{j}-\sum \mathfrak{q}_{j}+2 \mathfrak{q}_{k} \leq \ell_{k}+\ell_{k+1}+\mathfrak{q}_{k}
$$

Inequality (2.9) is satisfied for each $k$, since by hypothesis we have $\sum \ell\left(I_{j}\right)=\sum \ell_{j} \leq \pi$, and thus

$$
\sum \ell_{j}-\sum \mathfrak{q}_{j}=\sum \ell_{j}-\left(2 \pi-\sum \ell_{j}\right) \leq 0 .
$$

On the other hand, we claim that (2.10) holds for at least one $k$. Indeed, argue by contradiction: if $\sum \ell_{j}-\sum \mathfrak{q}_{j}+2 \mathfrak{q}_{k}>\ell_{k}+\ell_{k+1}+\mathfrak{q}_{k}$ for each $k$, then we obtain by summation that $(l-2) \sum \ell_{j}>(l-1) \sum \mathfrak{q}_{j}$. But this is impossible, since $\sum \ell_{j} \leq \sum \mathfrak{q}_{j}$.

The proof of Lemma 2.1 is complete. 


\section{Lower bounds for the energy of rotational mini- mizers}

Let $\Omega$ be an open set in $\mathbb{R}^{2}$. All results in this section apply to potentials of the form $F\left(x,|u|^{2}\right)$, where
a) $F: \Omega \times \mathbb{R}_{+} \rightarrow \mathbb{R}_{+}$.
b) $F(x, 1)=0, \forall x \in \Omega$.
c) $[0,1] \ni t \mapsto F(x, t)$ is non increasing, $\forall x \in \Omega$.
d) $F \in C^{1}(\bar{\Omega} \times[0,1])$.

Unless stated otherwise, in all the results in this section we assume that a)-d) hold.

The energy associated to $F$ is

$$
E(u)=\frac{1}{2} \int_{\Omega}|\nabla u|^{2}+\frac{1}{2} \int_{\Omega} F\left(x,|u|^{2}\right), \quad \forall u \in H^{1}(\Omega ; \mathbb{C}) .
$$

3.1 Examples. We have in mind two typical examples:

a) $F \equiv 0$, leading to harmonic functions.

b) $F(x, t)=\frac{1}{2 \varepsilon^{2}} w(x)(1-t)^{2}$, with $w \in C^{1}\left(\bar{\Omega} ; \mathbb{R}_{+}\right)$. This corresponds, e.g., to the standard Ginzburg-Landau potential (when $w \equiv 1$ ) possibly after a conformal change of variables (in which case $w$ is the Jacobian of the conformal representation).

3.2 Lemma. Let $F: \Omega \times \mathbb{R}_{+} \rightarrow \mathbb{R}_{+}$satisfy

a) $F(x, 1)=0, \forall x \in \Omega$.

b) $F \in C^{1}(\bar{\Omega} \times[0,1])$.

Let $\Omega$ be Lipschitz and let $g \in H^{1 / 2}(\partial \Omega ; \mathbb{C})$ satisfy $|g| \leq 1$. If $u$ minimizes $E$ in $H_{g}^{1}(\Omega ; \mathbb{C})$, then $|u| \leq 1$.

In particular, we have $u \in C_{\text {loc }}^{1, \alpha}(\Omega), 0<\alpha<1$.

Proof. We have $E(u) \leq E(\Pi \circ u)$, where $\Pi$ is the nearest point projection onto $\overline{\mathbb{D}}$. Since the differential of $\Pi$ satisfies

$$
|d \Pi(z) \xi|<|\xi|, \quad \forall \xi \in \mathbb{R}^{2} \backslash\{0\}, \forall z \in \mathbb{C} \backslash \overline{\mathbb{D}},
$$

we find that $\nabla u=0$ a.e. in the set $\{x \in \Omega ;|u(x)|>1\}$, so that $|u| \leq 1$ a.e.

The second part of the lemma follows from the boundedness of $-\Delta u$.

3.3 Definition. Let $\Omega \subset \mathbb{R}^{2}$ be a bounded $C^{1}$ domain. Let $\Gamma_{j}, j \in \llbracket 0, k \rrbracket$, be the components of $\partial \Omega$, with $\Gamma_{0}$ enclosing $\Omega$.

The map $u \in H^{1}(\Omega ; \mathbb{C})$ is a rotational minimizer ${ }^{8}$ if: for each $\alpha_{0}, \ldots, \alpha_{k} \in \mathbb{S}^{1}$ and for each $v \in H^{1}(\Omega ; \mathbb{C})$ such that $v=\alpha_{j} u$ on $\Gamma_{j}, j \in \llbracket 0, k \rrbracket$, we have $E(u) \leq E(v)$.

3.4 Remarks. In view of the specific form of the potential $F$, it is easy to see that:

a) When $\Omega$ is simply connected, rotational minimizer is the same as minimizer of $E$ with respect to its own boundary condition.

b) In general, the fact that $u$ is a rotational minimizer is equivalent to: if $v=\alpha_{j} u$ on $\Gamma_{j}$ with $j \geq 1$ (with $\alpha_{j}$ as above), then $E(u) \leq E(v)$.

\subsection{Examples.}

a) A prescribed degrees minimizer is a rotational minimizer.

\footnotetext{
${ }^{8}$ With respect to $E$, but this will not be specified.
} 
b) A more involved example: let $\Omega$ be simply connected. Let $u$ minimize $E$ with respect to its own boundary condition. Assume that $p<\underset{\partial \Omega}{\operatorname{essinf}}|u|$ is a regular value of $|u|$. Let $\omega=\{x \in \Omega ;|u(x)|<p\}$. Then $u$ is a rotational minimizer on $\omega$. To see this, we first note that $\bar{\omega} \subset \Omega$ (this follows from the proof of (3.3) below). Let $\gamma_{j}, j \in \llbracket 0, l \rrbracket$, be the components of $\partial \omega$, with $\gamma_{0}$ enclosing $\omega$. Let $v \in H^{1}(\omega)$ be such that $v=u$ on $\gamma_{0}, v=\alpha_{j} u$ on $\gamma_{j}, j \in \llbracket 1, l \rrbracket$, with $\alpha_{j} \in \mathbb{S}^{1}$. Let $\omega_{j}$ be the interior of $\gamma_{j}, j \in \llbracket 0, l \rrbracket$. Let

$$
w(x)=\left\{\begin{array}{ll}
u(x), & \text { in } \Omega \backslash \omega_{0} \\
v(x), & \text { in } \omega \\
\alpha_{j} u(x), & \text { if } x \in \omega_{j} \text { for some } j \geq 1
\end{array} .\right.
$$

The inequality $E(u) \leq E(w)$ (in $\Omega$ ) implies $E(u) \leq E(v)$ (in $\omega$ ).

c) Let $\Omega$ be simply connected. Let $u$ minimize $E$ with respect to its own boundary condition. Let $\omega \subset \Omega$ be a $C^{1}$ domain such that either $\bar{\omega} \subset \Omega$ or $\partial \Omega \subset \partial \omega$. Then $u$ is a rotational minimizer in $\omega$. The argument is the same as in the previous item.

3.6 Theorem. Let $u$ be a rotational minimizer. Assume that $t \leq|u| \leq 1$ on $\partial \Omega$. Let $s=\inf _{\Omega}|u|$. Then (with $\mathscr{A}_{s, t}$ as in Section $1.1 \mathrm{f}$ ))

$$
\int_{\Omega}|\nabla u|^{2} \geq 4 \mathscr{A}_{s, t}
$$

and in particular

$$
E(u) \geq 2 \mathscr{A}_{s, t} .
$$

3.7 Corollary. Let $u$ be a rotational minimizer. Assume that $|u|=1$ on $\partial \Omega$ and that $u$ vanishes at some point. Then $\int_{\Omega}|\nabla u|^{2} \geq 2 \pi$.

The conclusion of the corollary is optimal: take $\Omega=\mathbb{D}, F \equiv 0$ and $u(z) \equiv z$.

3.8 Remark. There is a simple proof of Corollary 3.7 when $F \equiv 0$ and $\Omega$ is simply connected. Indeed, after a conformal change of variables we may assume that $\Omega=\mathbb{D}$ and $u(0)=0$. Write, on $\mathbb{S}^{1}, u=\sum a_{n} e^{i n \theta}$. Condition $u(0)=0$ reads $a_{0}=0$. Condition $|u| \equiv 1$ on $\mathbb{S}^{1}$ implies $2 \pi=$ $\int_{\mathbb{S}^{1}}|u|^{2}=2 \pi \sum\left|a_{n}\right|^{2}$. On the other hand, it is easy to see that $\int_{\mathbb{D}}|\nabla u|^{2}=2 \pi \sum|n|\left|a_{n}\right|^{2}$. We find that

$$
\int_{\mathbb{D}}|\nabla u|^{2}=2 \pi \sum|n|\left|a_{n}\right|^{2} \geq 2 \pi \sum_{n \neq 0}\left|a_{n}\right|^{2}=2 \pi \sum\left|a_{n}\right|^{2}=2 \pi
$$

3.9 Remark. By Remark 3.4 a), in a simply connected domain Theorem 3.6 applies to a minimizer $u$ of $E$ with respect to its own boundary condition. However, if, in a multiply connected domain, we replace, in Theorem 3.6, the assumption that $u$ is a rotational minimizer with the weaker assumption that $u$ minimizes $E$ with respect to its own boundary condition, then the conclusion of Theorem 3.6 need not hold anymore. Here is an example. Let $\Omega=\mathbb{D} \backslash \overline{\mathbb{D}}_{t}$, with $0<t<1, F \equiv 0$ and $g(z)=\left\{\begin{array}{ll}1, & \text { if }|z|=1 \\ -1, & \text { if }|z|=t\end{array}\right.$. Then the minimizer of $E$ with datum $g$ is the harmonic extension of $g$, which vanishes on a circle. However, we have, by a direct calculation, $E(u) \rightarrow 0$ as $t \backslash 0$.

Proof of Theorem 3.6. We may assume that $s<t$, for otherwise there is nothing to prove. We start by noting that

$$
\lim _{\varepsilon \rightarrow 0} \inf \{|u(x)| ; x \in \Omega, \operatorname{dist}(x, \partial \Omega)<\varepsilon\} \geq t .
$$


This can be obtained (via some arguments developed in $[10,11])$ as follows. The map $g=u_{\mid \partial \Omega}$ is in $H^{1 / 2}$, and thus in VMO (since, in one dimension, we have $H^{1 / 2} \hookrightarrow$ VMO [10, Example 2, p. 201]). Since $g$ is in VMO and takes its values into the closed set $F=\{z \in \mathbb{C} ;|z| \geq t\}$, it follows that for $x$ near $\partial \Omega$ the harmonic extension $\tilde{g}$ of $g$ takes its values near $F$ [11, Theorem A.3.2, p. 357]; in particular, (3.3) holds when $u$ is replaced with $\tilde{g}$. On the other hand the map $u-\tilde{g}$ vanishes on $\partial \Omega$ and (using the equation satisfied by $u$ ) belongs to $C^{1, \alpha}(\bar{\Omega})$ for each $0<\alpha<1$. This easily implies (3.3).

We find that, for $0<s \leq m<t$, the level lines $\mathscr{C}_{m}=\{x \in \Omega ;|u(x)|=m\}$ are compact. Since $u \in C^{1}(\Omega)$, Sard's lemma implies that, for a.e. $m, \mathscr{C}_{m}$ is a finite union of $C^{1}$ disjoint simple closed curves.

We fix a point $x_{0} \in \Omega$ such that $\left|u\left(x_{0}\right)\right|=s$.

3.10 Lemma. Let $s<m<t$ be a regular value of $|u|$. Let $\omega$ be the connected component of the set $\{x \in \Omega ;|u(x)|<m\}$ containing $x_{0}$. Then:

Either

a) $\ell(u(\partial \omega))>\pi m$.

b) There is some contraction $\mathbb{P}$ preserving the modulus such that $v=\mathbb{P} \circ u$ is a rotational minimizer and such that $v(\partial \omega)$ is connected.

Proof of Lemma 3.10. We may write $\partial \omega=\gamma_{0} \cup \ldots \cup \gamma_{l}$, where the components $\gamma_{j}$ are disjoint and $\gamma_{0}$ encloses $\omega$. Assume that $\ell(u(\partial \omega)) \leq \pi m$. Since $u(\partial \omega) \subset C_{m}$, Lemma 2.1 applied to the arcs $u\left(\gamma_{j}\right), j \in \llbracket 0, l \rrbracket$, implies the existence of a contraction $\mathbb{P}: \mathbb{R}^{2} \rightarrow \mathbb{R}^{2}$ such that $\mathbb{P}$ preserves the modulus and such that $\mathbb{P}$ is a rotation on each $u\left(\gamma_{j}\right)$. We may clearly assume that $\mathbb{P}$ restricted to $u\left(\gamma_{0}\right)$ is the identity. Let, for $j \geq 1, \mathscr{R}_{j}$ be a rotation satisfying $\mathbb{P}=\mathscr{R}_{j}$ on $u\left(\gamma_{j}\right)$. Let, for $j \geq 0$, $\omega_{j}$ be the intersection of $\Omega$ with the interior of $\gamma_{j}$. Set

$$
v(x)=\left\{\begin{array}{ll}
u(x), & \text { if } x \in \bar{\Omega} \backslash \omega_{0} \\
\mathbb{P}(u(x)), & \text { if } x \in \omega \\
\mathscr{R}_{j}(u(x)), & \text { if } x \in \omega_{j} \text { for some } j \geq 1
\end{array} .\right.
$$

Let $\Gamma_{n}, n \in \llbracket 0, k \rrbracket$, be the components of $\partial \Omega$. Then, for each $n$, there is some $\alpha_{n} \in \mathbb{S}^{1}$ such that $v=\alpha_{n} u$ on each $\Gamma_{n}$. In addition, we clearly have $\int_{\Omega} F\left(x,|v|^{2}\right)=\int_{\Omega} F\left(x,|u|^{2}\right)$ and $\int_{\Omega}|\nabla v|^{2} \leq$ $\int_{\Omega}|\nabla u|^{2}$. Therefore, $E(v) \leq E(u)$. To summarize: on each component $\Gamma_{n}$ of $\partial \Omega$, we have $v=\alpha_{n} u$, with $\alpha_{n} \in \mathbb{S}^{1}$, and $E(v) \leq E(u)$. Since $u$ is a rotational minimizer, we find that so is $v$. Finally, $v(\partial \omega)$ is connected by construction.

3.11 Lemma. Let $s<m<t$ be a regular value of $|u|$. Then

$$
\int_{\{|u|=m\}}|\nabla(u /|u|)| \geq 2 \ell_{s, m} / m
$$

Recall that $\ell_{s, m}$ is the length of $C_{m} \cap\{\operatorname{Re} z>s\}$.

Proof of Lemma 3.11. We use the notation in the statement and proof of Lemma 3.10. Let $\omega$ be the connected component of the set $\{x \in \Omega ;|u(x)|<m\}$ containing $x_{0}$. We write $\partial \omega=\gamma_{0} \cup \ldots \cup \gamma_{l}$. We start by proving that

$$
\int_{\gamma_{j}}|\nabla(u /|u|)| \geq \int_{\gamma_{j}}\left|\partial_{\tau}(u /|u|)\right|=\frac{1}{m} \int_{\gamma_{j}}\left|\partial_{\tau} u\right| \geq 2 \ell\left(u\left(\gamma_{j}\right)\right) / m ;
$$

here, $\partial_{\tau}$ stands for the tangential derivative on $\gamma_{j}$. 
(3.5) is obtained as follows. Let $A, B \in \gamma_{j}$ be such that the endpoints of $u\left(\gamma_{j}\right)$ are $u(A)$ and $u(B){ }^{9}$ Let $\mathscr{C}_{p}, p=1,2$, be the two arcs of $\gamma_{j}$ with endpoints $A$ and $B$. Write, on each $\mathscr{C}_{p}$, $u=m e^{\imath \varphi_{p}}$. Since $\left|\varphi_{p}(A)-\varphi_{p}(B)\right|=\ell\left(u\left(\gamma_{j}\right)\right) / m, p=1,2$, we find that

$$
\int_{\gamma_{j}}\left|\partial_{\tau}(u /|u|)\right|=\sum_{p} \int_{\mathscr{C}_{p}}\left|\partial_{\tau} \varphi_{p}\right| \geq \sum_{p}\left|\varphi_{p}(A)-\varphi_{p}(B)\right| \geq 2 \ell\left(u\left(\gamma_{j}\right)\right) / m .
$$

In particular, the conclusion of Lemma 3.11 holds if $\ell(u(\partial \omega))>\pi m$.

Consider now the case where $\ell(u(\partial \omega)) \leq \pi m$. Let $v$ be as in Lemma 3.10. Since $v=\mathbb{P} \circ u$, with $\mathbb{P}$ contraction preserving the modulus, we find that

$$
\int_{\{|u|=m\}}|\nabla(u /|u|)| \geq \int_{\{|u|=m\}}\left|\partial_{\tau}(u /|u|)\right| \geq \int_{\{|u|=m\}}\left|\partial_{\tau}(v /|v|)\right|=\int_{\{|v|=m\}}\left|\partial_{\tau}(v /|v|)\right|,
$$

so that it suffices to prove that

$$
\int_{\partial \omega}\left|\partial_{\tau}(v /|v|)\right| \geq 2 \ell_{s, m} / m
$$

This is proved by contradiction. Assume that

$$
\int_{\partial \omega}\left|\partial_{\tau}(v /|v|)\right|<2 \ell_{s, m} / m
$$

By combining (3.5) (applied to $v$ ) with (3.7) and with the fact that $v(\partial \omega)$ is connected, we find that $v(\partial \omega)$ is contained in an $\operatorname{arc} \mathscr{C} \subset C_{m}$ of length $<\ell_{s, m}$. Without loss of generality, we may assume that $\mathscr{C}$ is centered at $m .{ }^{10}$ Condition $\ell(\mathscr{C})<\ell_{s, m}$ implies that there exists some $q \in(s, m)$ such that

$$
\mathscr{C} \subset S=\{z \in \overline{\mathbb{D}} ; \operatorname{Re} z \geq q\} .
$$

We next note that $v$ is, in $\omega$, a minimizer of $E$ with respect with its own boundary condition, and that $\left|v\left(x_{0}\right)\right|=s$, so that $v\left(x_{0}\right) \notin S$. Property $v\left(x_{0}\right) \notin S$ combined with (3.8) contradicts our next result. This completes the proof of Lemma 3.11 .

3.12 Lemma. Let $S$ be as in (3.8). Let u minimize $E$ in some Lipschitz domain $\omega$ with respect with its own boundary datum $g \in H^{1 / 2} \cap C^{0}(\partial \omega$; $\mathbb{C})$. If $g(\partial \omega) \subset S$, then $u(\omega) \subset S$.

Special cases of this result appear in [2, Lemma 8] and [13, Lemma 2]. However, the arguments there do not cover the case $F \equiv 0$.

Proof of Lemma 3.12. We may assume that $\omega$ is connected. By Lemma 3.2, we have $|u| \leq 1$ in $\omega$ and $u$ is continuous in $\omega$.

Let $g=u_{\mid \partial \omega}$. The map $v=|\operatorname{Re} u|+\imath \operatorname{Im} u$ equals $g$ on $\partial \omega$ and has the same energy as $u$. Thus $v$ minimizes $E$. In particular, $v$ is continuous. Therefore, if we prove that $v(\omega) \subset S$, then we will also have $u(\omega) \subset S$. In conclusion, we reduced the problem to the case where $\operatorname{Re} u \geq 0$.

Let $\Pi$ be the orthogonal projection on $S$. When $z \in \overline{\mathbb{D}} \cap\{\operatorname{Re} z \geq 0\}$, we have (with $q$ given by (3.8))

$$
\Pi(z)= \begin{cases}z, & \text { if } \operatorname{Re} z \geq q \\ q+\imath \operatorname{Im} z, & \text { if }|\operatorname{Im} z| \leq \sqrt{1-q^{2}} \text { and } \operatorname{Re} z<q . \\ q+\imath(\operatorname{sgn} \operatorname{Im} z) \sqrt{1-q^{2}}, & \text { if }|\operatorname{Im} z|>\sqrt{1-q^{2}} \text { and } \operatorname{Re} z<q\end{cases}
$$

The following inequality is straightforward and left to the reader.

$$
|z| \leq|\Pi(z)| \leq 1 \text { for } z \in \overline{\mathbb{D}} \cap\{\operatorname{Re} z \geq 0\} .
$$

\footnotetext{
${ }^{9}$ The argument developed below holds under the assumption that each $u\left(\gamma_{j}\right)$ is strictly included in $C_{m}$; indeed, $u\left(\gamma_{j}\right)$ is supposed to have endpoints. In the case where one of these arcs, say $u\left(\gamma_{0}\right)$, coincides with $C_{m}$, the proof of (3.5) yields the inequality $\int_{\gamma_{0}}|\nabla(u /|u|)| \geq 2 \pi$. In this case, the conclusion of Lemma 3.11 is clear.

${ }^{10}$ Here, $m$ is identified with the complex number $m+\imath 0$.
} 
Set $w=\Pi \circ u$, which equals $g$ on $\partial \omega$. Since $\Pi$ is a contraction, we have $|\nabla w| \leq|\nabla u|$. This fact combined with (3.10), with the fact that $u$ minimizes $E$, and with property c) satisfied by the potential $F$, implies that $w$ is a minimizer of $E$ with boundary data $g$. Actually, a bit more can be said. Indeed, by combining the minimality of $u$ with the fact that $|\nabla w| \leq|\nabla u|$ and with the inequality $F\left(x,|w|^{2}\right) \leq F\left(x,|u|^{2}\right)$, we come up with the equalities $|\nabla w|=|\nabla u|$ and $F\left(x,|w|^{2}\right)=F\left(x,|u|^{2}\right) .^{11}$

Consider now the open set

$$
V=\{x \in \omega ; u(x) \notin S\}=\{x \in \omega ; \operatorname{Re} u<q\} .
$$

Our aim is to prove that $V$ is empty. Let $\Pi=\Pi_{1}+\imath \Pi_{2}$ and set

$$
F=\{z \in \overline{\mathbb{D}} \backslash S ; \operatorname{Re} z \geq 0\} .
$$

If $z \in F$, then $\Pi_{1}(z)=q$ and $\Pi_{2}(z)$ depends only on $\operatorname{Im} z$. Moreover, it is clear, from the specific form of $\Pi_{2}$, that

$$
\left|\Pi_{2}(z)-\Pi_{2}(\xi)\right| \leq|\operatorname{Im} z-\operatorname{Im} \xi|, \quad \forall z, \xi \in F .
$$

This implies at once that $\nabla(\operatorname{Re} w)=0$ and $|\nabla(\operatorname{Im} w)| \leq|\nabla(\operatorname{Im} u)|$ a.e. in $V$. These facts combined with the property $|\nabla w|=|\nabla u|$ a.e. imply $\nabla(\operatorname{Re} u)=0$ a.e. in $V$, and thus $\operatorname{Re} u$ is locally constant in $V$.

Assuming, by contradiction, that $V$ is not empty, we claim that $\operatorname{Re} u=q$ on $\partial V$. Indeed, let $V_{0}$ be a connected component of $V$. If $x \in \partial V_{0}$, then either $u(x) \in S$, or $x \in \partial \omega$. In the first case, the definition of $V$ implies $\operatorname{Re} u(x)=q$; in the latter, we obtain the same conclusion via the fact that $g \in C^{0}(\partial \omega ; \mathbb{C})$. Since $\operatorname{Re} u$ is locally constant in $V$, we find that $\operatorname{Re} u=q$ in $V$. This contradiction completes the proof of Lemma (3.12).

Proof of Theorem 3.6 completed. Write, locally in the set $U=\{x \in \Omega ; u(x) \neq 0\}, u$ in the form $u=\rho e^{\imath \varphi}$, with $\rho=|u|$ and $\varphi$ real-valued and $C^{1}$. On the one hand, we have

$$
|\nabla u|^{2}=|\nabla \rho|^{2}+\rho^{2}|\nabla \varphi|^{2} \geq 2 \rho|\nabla \varphi||\nabla \rho| \quad \text { in } U .
$$

On the other hand, the conclusion of Lemma 3.11 can be written as

$$
\int_{\{\rho=m\}}|\nabla \varphi| \geq 2 \ell_{s, m} / m \text {. }
$$

By combining (3.11) with (3.12) and applying twice the co-area formula, we find that

$$
\frac{1}{2} \int_{\Omega}|\nabla u|^{2} \geq \int_{U} \rho|\nabla \varphi||\nabla \rho|=\int_{s}^{t}\left(\int_{\{\rho=m\}} m|\nabla \varphi|\right) d m \geq 2 \int_{s}^{t} \ell_{s, m} d m=2 \mathscr{A}_{s, t} .
$$

\section{Wente estimates and applications}

Throughout this section, $\Omega$ is a $C^{1}$ bounded domain in $\mathbb{R}^{2}$.

The standard Wente estimates [25] apply to solutions $u$ vanishing at infinity of the equation $-\Delta u=\operatorname{Jac}(g, h)$ in $\mathbb{R}^{2}$. A sharp form of these estimates in domains has been found by Bethuel and Ghidaglia [6] and is recalled below.

4.1 Theorem ([6]). Let $f \in H_{0}^{1}(\Omega), g, h \in H^{1}(\Omega)$. Let $u \in W_{0}^{1,1}(\Omega)$ be the solution of $-\Delta u=$ $\operatorname{Jac}(g, h)$. Then

a) $u \in L^{\infty}(\Omega)$ and

$$
\|u\|_{L^{\infty}} \leq 2\|\nabla g\|_{L^{2}}\|\nabla h\|_{L^{2}} .
$$

\footnotetext{
${ }^{11}$ All these equalities are to be understood in the a.e. sense.
} 
b) $u \in H_{0}^{1}(\Omega)$ and

$$
\|\nabla u\|_{L^{2}} \leq \sqrt{2}\|\nabla g\|_{L^{2}}\|\nabla h\|_{L^{2}}
$$

c) We have

$$
\left|\int_{\Omega} f \operatorname{Jac}(g, h)\right| \leq \sqrt{2}\|\nabla f\|_{L^{2}}\|\nabla g\|_{L^{2}}\|\nabla h\|_{L^{2}} .
$$

As a consequence of (4.3), the map

$$
\left(H^{1}(\Omega)\right)^{2} \ni(g, h) \mapsto \operatorname{Jac}(g, h) \in H^{-1}(\Omega)
$$

is continuous. A stronger property is given by the following

4.2 Lemma. Let $g \in H^{1}(\Omega)$. Then the map

$$
H^{1}(\Omega) \ni h \mapsto \operatorname{Jac}(g, h) \in H^{-1}(\Omega)
$$

is compact.

Proof. Compactness from $H^{1}(\Omega)$ into $\mathscr{D}^{\prime}(\Omega)$ is a special case compensated compactness [21, Théorème 1]. However, compactness from $H^{1}(\Omega)$ into $H^{-1}(\Omega)$ requires an additional argument. We have to prove that, if $h_{n} \rightarrow 0$ in $H^{1}(\Omega)$, then $\operatorname{Jac}\left(g, h_{n}\right) \rightarrow 0$ in $H^{-1}(\Omega)$. Equivalently, if $u_{n} \in H_{0}^{1}(\Omega)$ solves $-\Delta u_{n}=\operatorname{Jac}\left(g, h_{n}\right)$, we have to prove that $u_{n} \rightarrow 0$ in $H^{1}(\Omega)$. By compensated compactness, we know that $u_{n} \rightarrow 0$ in $\mathscr{D}^{\prime}(\Omega)$. Since, on the one hand, $\left(u_{n}\right)$ is bounded in $H_{0}^{1}(\Omega)$ (by (4.2)) and, on the other hand, $u_{n} \rightarrow 0$ in $\mathscr{D}^{\prime}(\Omega)$, the Rellich-Kondratchov theorem implies that $u_{n} \rightarrow 0$ in $L^{2}(\Omega)$. By (4.1), we have $\left|u_{n}\right| \leq C$. Consequently, we have $u_{n} \nabla g \rightarrow 0$ in $L^{2}(\Omega)$. We find that

$$
\int_{\Omega}\left|\nabla u_{n}\right|^{2}=\int_{\Omega} u_{n}\left(-\Delta u_{n}\right)=\int_{\Omega}\left(u_{n} \nabla g\right) \wedge \nabla h_{n} \rightarrow 0,
$$

so that $u_{n} \rightarrow 0$ in $H^{1}(\Omega)$.

The above lemma is useful in establishing a crucial estimate, Lemma 4.4 below. For the convenience of the reader, we start by stating and proving a baby estimate, Lemma 4.3 below. Lemma 4.4 is a more involved variant of Lemma 4.3.

4.3 Lemma. Let $\left(g_{n}\right),\left(h_{n}\right) \subset H^{1}(\Omega)$. Assume that $g_{n} \rightarrow g$ in $H^{1}(\Omega)$. Let $\left(a_{n}\right)$ be a sequence of bounded nonnegative measurable functions in $\Omega$ satisfying $a_{n} \rightarrow \infty$ a.e. Let $u_{n} \in H_{0}^{1}(\Omega)$ be the solution of

$$
-\Delta u_{n}+a_{n} u_{n}=\operatorname{Jac}\left(g_{n}, h_{n}\right)
$$

Then

$$
\left\|\nabla u_{n}\right\|_{L^{2}}=o\left(\left\|\nabla h_{n}\right\|_{L^{2}}\right) \quad \text { as } n \rightarrow \infty .
$$

Proof. We may assume that $\left\|\nabla h_{n}\right\|_{L^{2}}=1$; in this case, we have to prove that $\left\|\nabla u_{n}\right\|_{L^{2}} \rightarrow 0$. By a standard argument, it suffices to establish this property along a subsequence. Therefore, we may further assume that $h_{n} \rightarrow h$ in $H^{1}(\Omega)$. Let $F_{n}=\operatorname{Jac}\left(g_{n}, h_{n}\right)$ and $F=\operatorname{Jac}(g, h)$. By (4.2) and Lemma 4.2 , we have $F_{n} \rightarrow F$ in $H^{-1}(\Omega)$.

If we multiply (4.4) by $u_{n}$ and use (4.3), we find that

$$
\int_{\Omega}\left|\nabla u_{n}\right|^{2} \leq \int_{\Omega} u_{n} F_{n} \leq \sqrt{2}\left\|\nabla u_{n}\right\|_{L^{2}}\left\|\nabla g_{n}\right\|_{L^{2}}\left\|\nabla h_{n}\right\|_{L^{2}},
$$


so that $\left(u_{n}\right)$ is bounded in $H^{1}(\Omega)$. On the other hand, we have (again, by multiplying (4.4) by $\left.u_{n}\right) \int_{\Omega} a_{n} u_{n}^{2} \leq \int_{\Omega} u_{n} F_{n}$, so that the quantity $\int_{\Omega} a_{n} u_{n}^{2}$ is uniformly bounded. We find that $\int_{\Omega} u_{n}^{2} \rightarrow 0 .^{12}$

To summarize: we proved that (up to a subsequence) $u_{n} \rightarrow 0$ in $H^{1}(\Omega)$. Finally, we have

$$
\int_{\Omega}\left|\nabla u_{n}\right|^{2} \leq \int_{\Omega} u_{n} F_{n} \rightarrow 0
$$

(since $u_{n} \rightarrow 0$ in $H^{1}(\Omega)$ and $F_{n} \rightarrow F$ in $H^{-1}(\Omega)$ ), so that $u_{n} \rightarrow 0$ in $H^{1}(\Omega)$.

4.4 Lemma. Let $g_{n}, h_{n}, a_{n}$ be as in Lemma 4.3. Assume that $v_{n} \in H_{0}^{1}(\Omega)$ satisfies

$$
-\Delta v_{n}+a_{n} v_{n}=\operatorname{Jac}\left(g_{n}, h_{n}\right)+\operatorname{div} R_{n}+v_{n} S_{n}+T_{n},
$$

where

$$
\left\|R_{n}\right\|_{L^{2}}=o\left(\left\|\nabla v_{n}\right\|_{L^{2}}\right),\left\|S_{n}\right\|_{L^{2}}=O\left(\left\|\nabla h_{n}\right\|_{L^{2}}\right),\left\|T_{n}\right\|_{L^{1}}=O\left(\left\|\nabla h_{n}\right\|_{L^{2}}^{2}\right) .
$$

Assume, in addition,

$$
v_{n} \rightarrow 0 \quad \text { uniformly in } \Omega \text { as } n \rightarrow \infty \text {. }
$$

Then

$$
\left\|\nabla v_{n}\right\|_{L^{2}}=o\left(\left\|\nabla h_{n}\right\|_{L^{2}}\right) \quad \text { as } n \rightarrow \infty .
$$

Proof. We start as in the proof of Lemma 4.3: we assume that $h_{n} \rightarrow h$, and let $F_{n}=\operatorname{Jac}\left(g_{n}, h_{n}\right)$, $F=\operatorname{Jac}(g, h)$. If we multiply (4.5) by $v_{n}$, we find that

$$
\int_{\Omega}\left|\nabla v_{n}\right|^{2} \leq \int_{\Omega} F_{n} v_{n}-\int_{\Omega} R_{n} \cdot \nabla v_{n}+\left\|v_{n}\right\|_{L^{\infty}}^{2} \int_{\Omega}\left|S_{n}\right|+\left\|v_{n}\right\|_{L^{\infty}} \int_{\Omega}\left|T_{n}\right| .
$$

Using (4.6) and (4.7), we find that $\left(v_{n}\right)$ is bounded in $H^{1}(\Omega)$. Since (using again (4.5)) we have

$$
\int_{\Omega} a_{n} v_{n}^{2} \leq \int_{\Omega} F_{n} v_{n}-\int_{\Omega} R_{n} \cdot \nabla v_{n}+\left\|v_{n}\right\|_{L^{\infty}}^{2} \int_{\Omega}\left|S_{n}\right|+\left\|v_{n}\right\|_{L^{\infty}} \int_{\Omega}\left|T_{n}\right|,
$$

we find that $\int_{\Omega} a_{n} v_{n}^{2}$ is bounded uniformly in $n$, and thus (as in the proof of Lemma 4.3) $v_{n} \rightarrow 0$ in $H^{1}(\Omega)$. Using (4.8), we obtain that $v_{n} \rightarrow 0$ in $H^{1}(\Omega){ }^{13}$

\section{Degree, lifting, and the Ginzburg-Landau equation}

For the convenience of the reader, we gather here some known facts used in the proofs of Theorems 1.2 and 1.5. The references for this section are: for the degree, [10], [11], [8], [9]; for lifting, [7], [9]; for GL, [4], [16], [1].

Let $\Gamma$ be a smooth simple planar curve. A map $g \in H^{1 / 2}\left(\Gamma ; \mathbb{S}^{1}\right)$ has a well-defined degree (winding number), denoted $\operatorname{deg}(g, \Gamma)$. This degree is defined as follows: smooth maps are dense in $H^{1 / 2}\left(\Gamma ; \mathbb{S}^{1}\right)$, and the degree is continuous with respect the $H^{1 / 2}$ convergence. Thus we may define

$$
\operatorname{deg}(g, \Gamma)=\lim \operatorname{deg}\left(g_{n}, \Gamma\right)
$$

\footnotetext{
${ }^{12}$ The abstract fact we use is the following: if $\left(u_{n}\right)$ is compact in $L^{2}, a_{n} \geq 0$ and $a_{n} \rightarrow \infty$ a.e., and if $\int a_{n} u_{n}^{2}$ is bounded, then $u_{n} \rightarrow 0$ in $L^{2}$.

${ }^{13}$ Here, we use: $v_{n} \rightarrow 0$ in $H^{1}(\Omega)$ and $F_{n} \rightarrow F$ in $H^{-1}(\Omega)$ for the first integral on the right-hand side, $v_{n} \rightarrow 0$ in $H^{1}(\Omega)$ for the second one, and $\left\|v_{n}\right\|_{L^{\infty}} \rightarrow 0$ combined with (4.6) for the two last ones.
} 
for some sequence $\left(g_{n}\right) \subset C^{\infty}\left(\Gamma ; \mathbb{S}^{1}\right)$ such that $g_{n} \rightarrow g$ dans $H^{1 / 2}$. The degree defined in this way does not depend on the choice of the sequence $\left(g_{n}\right)$ and is continuous with respect to the $H^{1 / 2}$ convergence.

The degree of $H^{1 / 2}$ maps inherits some well-known properties of the degree of continuous maps:

a) $\operatorname{deg}(g h, \Gamma)=\operatorname{deg}(g, \Gamma)+\operatorname{deg}(h, \Gamma)$.

b) $\operatorname{deg}(g / h, \Gamma)=\operatorname{deg}(g, \Gamma)-\operatorname{deg}(h, \Gamma)$.

c) $\operatorname{deg}(g, \Gamma)=0 \Longleftrightarrow g=e^{\imath \varphi}$ for some $\varphi \in H^{1 / 2}(\Gamma ; \mathbb{R})$.

Moreover,

d) If $g \in C \cap H^{1 / 2}\left(\Gamma ; \mathbb{S}^{1}\right)$, then the degree of $g$ in the sense $H^{1 / 2}$ maps is the same as the degree of $g$ in the sense of continuous maps.

We consider, for $H^{1 / 2}$ maps defined on $\Gamma$, the following semi-norm:

$$
|g|_{H^{1 / 2}}^{2}=\int_{\Omega}|\nabla u|^{2}
$$

here, $\Omega$ is the interior of $\Gamma$ and $u$ is the harmonic extension of $g$. This semi-norm is invariant with respect to conformal representations; therefore, we will consider, in what follows, only the case $\Gamma=\mathbb{S}^{1}$ and $\Omega=\mathbb{D}$; the general case reduces to this one, by the Riemann mapping theorem.

If $g: \mathbb{S}^{1} \rightarrow \mathbb{C}$, and if we write $g=\sum a_{n} e^{i n \theta}$, then

e) $|g|_{H^{1 / 2}}^{2}=2 \pi \sum|n|\left|a_{n}\right|^{2}$.

f) If, in addition, $g$ is $\mathbb{S}^{1}$-valued, then $\operatorname{deg}\left(g, \mathbb{S}^{1}\right)=\sum n\left|a_{n}\right|^{2}$.

In particular, if $|g|_{H^{1 / 2}}^{2}<2 \pi$, then $\operatorname{deg}(g, \Gamma)=0$, and thus we may write $g=e^{\imath \varphi}$ for some $\varphi \in$ $H^{1 / 2}(\Gamma ; \mathbb{R})$. The next property asserts that, when $|g|_{H^{1 / 2}}$ is sufficiently small, we may control $\varphi$.

g) There is some $\delta>0$ such that, if $|g|_{H^{1 / 2}}<\delta$, then we may write $g=e^{\imath \varphi}$, with $|\varphi|_{H^{1 / 2}} \leq$ $C|g|_{H^{1 / 2}}$.

h) In particular, if $|g|_{H^{1 / 2}}<\delta$, then $g$ has an extension $v \in H^{1}\left(\Omega ; \mathbb{S}^{1}\right)$ such that $\int_{\Omega}|\nabla v|^{2} \leq$ $C^{2} \delta^{2} \cdot{ }^{14}$

Another important property of $\mathbb{S}^{1}$-valued maps is the following:

i) Let $\Omega \subset \mathbb{R}^{2}$ be a $C^{1}$ bounded domain. Let $g \in H^{1 / 2}\left(\partial \Omega ; \mathbb{S}^{1}\right)$. Let $u$ be the harmonic extension of $g$. Then

$$
\lim _{x \rightarrow \partial \Omega}|u(x)|=1 \quad \text { uniformly in } x .
$$

The same property holds for a minimizer of $E_{\varepsilon}$ with boundary datum $g$ or, more generally, for solutions of

$$
\left\{\begin{array}{ll}
\Delta u \in L^{\infty}(\Omega) & \text { in } \Omega \\
u=g & \text { on } \partial \Omega
\end{array}\right. \text {. }
$$

We next consider $\mathbb{S}^{1}$-valued maps in domains. Let $u \in H^{1}\left(\Omega ; \mathbb{S}^{1}\right)$, where $\Omega$ is a bounded domain in $\mathbb{R}^{2}$. Then we may write, locally in $\Omega, u=e^{\imath \varphi}$, with $\varphi \in H^{1}$. If $\Omega$ is simply connected, then we may choose a global phase $\varphi$. However, when $\Omega$ is multiply connected, it is not always possible to pick a global $\varphi$. As in the case of continuous maps, we have the following equivalence:

j) Let $\Omega \subset \mathbb{R}^{2}$ be a $C^{1}$ bounded domain. Let $u \in H^{1}\left(\Omega ; \mathbb{S}^{1}\right)$. We may write, globally in $\Omega$, $u=e^{\imath \varphi}$, with $\varphi \in H^{1}(\Omega ; \mathbb{R})$, if and only if $\operatorname{deg}(u, \Gamma)=0$ for each component $\Gamma$ of $\partial \Omega .{ }^{15} 16$

\footnotetext{
${ }^{14}$ Indeed, consider $v=e^{\imath \psi}$, where $\psi$ is the harmonic extension of $\varphi$.

${ }^{15}$ Note that $u_{\mid \Gamma} \in H^{1 / 2}\left(\Gamma ; \mathbb{S}^{1}\right)$, so that $\operatorname{deg}(u, \Gamma)$ makes sense.

${ }^{16}$ In the special case where $\Omega$ is simply connected, this condition is always satisfied, and the global lifting $\varphi$ exists.
} 
Similar results hold for maps which are far away from zero. More specifically, consider a map $u \in H^{1}(\Omega ; \mathbb{C})$ such that

$$
0<\alpha \leq|u| \leq \beta<\infty \quad \text { in } \Omega \text {. }
$$

Then we may write, locally in $\Omega, u=|u| e^{\imath \varphi}$, with $\varphi \in H^{1}$. In this context, the analog of $\mathrm{j}$ ) is given by

k) Let $\Omega \subset \mathbb{R}^{2}$ be a $C^{1}$ bounded domain. Let $u$ satisfy (5.3). We may write, globally in $\Omega$, $u=|u| e^{\imath \varphi}$, with $\varphi \in H^{1}(\Omega ; \mathbb{R})$, if and only if $\operatorname{deg}(u /|u|, \Gamma)=0$ for each component $\Gamma$ of $\partial \Omega$.

In simply connected domains, there is a connection between phases of $\mathbb{S}^{1}$-valued maps and phases of their traces.

1) Let $\Omega \subset \mathbb{R}^{2}$ be a $C^{1}$ bounded simply connected domain. Let $u \in H^{1}\left(\Omega ; \mathbb{S}^{1}\right)$ and set $g=u_{\mid \partial \Omega} \in$ $H^{1 / 2}\left(\partial \Omega ; \mathbb{S}^{1}\right)$. Write $u=e^{\imath \varphi}$ and $g=e^{l \varphi_{0}}$, where $\varphi \in H^{1}$ and $\varphi_{0} \in H^{1 / 2}$. Then $\varphi_{\mid \partial \Omega}=\varphi_{0}$ modulo a constant multiple of $2 \pi$. Similar result if we assume (5.3).

Easy consequences of this fact are given in the next item.

m) Let $\Omega$ be smooth bounded simply connected. Let $g \in H^{1 / 2}\left(\partial \Omega ; \mathbb{S}^{1}\right)$. Write $g=e^{\imath \varphi_{0}}$, where $\varphi_{0} \in H^{1 / 2}$. Consider the class

$$
\mathscr{C}=\left\{u \in H^{1}\left(\Omega ; \mathbb{S}^{1}\right), u_{\mid \partial \Omega}=g\right\} .
$$

Then

$$
\mathscr{C}=\left\{e^{\imath \varphi} ; \varphi \in H^{1}(\Omega ; \mathbb{R}), \varphi_{\mid \partial \Omega}=\varphi_{0}\right\} .
$$

If, in addition, $\Phi$ is the harmonic extension of $\varphi_{0}$, then $u_{0}=e^{\imath \Phi}$ is the only minimizer of

$$
\mathscr{C} \ni u \mapsto \int_{\Omega}|\nabla u|^{2} .
$$

We next turn to the question of the form of the GL equation in polar coordinates.

Consider first the case of Dirichlet boundary condition in a simply connected domain, with boundary datum $g$ of zero degree. If $u$ is a critical point of $E_{\varepsilon}$ such that essinf $|u|>0$, then we may write (globally) $u=\rho e^{\imath \varphi}$, with $\rho=|u|$ and $\varphi \in H^{1}$. In addition, we may write $g=e^{\imath \varphi_{0}}$, with $\varphi_{0} \in H^{1 / 2}(\partial \Omega ; \mathbb{R})$, and then (possibly after subtracting a suitable multiple of $2 \pi$ from $\varphi$ ) we have the system

$$
\left\{\begin{array}{ll}
\operatorname{div}\left(\rho^{2} \nabla \varphi\right)=0 & \text { in } \Omega \\
-\Delta \rho=\frac{1}{\varepsilon^{2}} \rho\left(1-\rho^{2}\right)-\rho|\nabla \varphi|^{2} & \text { in } \Omega \\
\rho=1 & \text { on } \partial \Omega \\
\varphi=\varphi_{0} & \text { on } \partial \Omega
\end{array} .\right.
$$

The first equation, together with the fact that $\Omega$ is simply connected, allows to define a potential $H$ satisfying

$$
\begin{aligned}
& \frac{\partial H}{\partial x}=\rho^{2} \frac{\partial \varphi}{\partial y}, \frac{\partial H}{\partial y}=-\rho^{2} \frac{\partial \varphi}{\partial x}, \quad \int_{\Omega} H=0, \\
& \operatorname{div}\left(\frac{1}{\rho^{2}} \nabla H\right)=0 .
\end{aligned}
$$

Consider now a second critical point, $v$, satisfying also essinf $|v|>0$. Then we may write $v=$ $\rho \eta e^{\imath(\varphi+\psi)}$, where $\eta=|v| /|u|, w=1-\eta$ and $\psi$ satisfy

$$
\left\{\begin{array}{ll}
\operatorname{div}\left(\rho^{2} \eta^{2} \nabla \psi\right)=\operatorname{div}\left(\rho^{2}\left(1-\eta^{2}\right) \nabla \varphi\right) & \text { in } \Omega \\
-\Delta w+a w=\operatorname{Jac}(2 H, w)+\operatorname{div} R+w S+T & \text { in } \Omega \\
\psi=0 & \text { on } \partial \Omega \\
w=0 & \text { on } \partial \Omega
\end{array} ;\right.
$$


here,

$$
a=\frac{1}{\varepsilon^{2}} \rho^{4} \eta(1+\eta), R=-\left(1-\rho^{2}\right) \nabla w, S=-2 \rho^{2} \nabla \varphi \cdot \nabla \psi, T=\rho^{2} \eta|\nabla \psi|^{2} .
$$

We next present the analogs of (5.4)-(5.8) in the case of prescribed degrees in a (possibly multiply connected) domain. If $u$ is a non vanishing critical point, then it may not be possible to write, globally, $u=\rho e^{\imath \varphi}$. However, the vector field $\nabla \varphi=(u /|u|) \wedge \nabla(u /|u|)$ is globally-defined, and (5.4) becomes

$$
\left\{\begin{array}{ll}
\operatorname{div}\left(\rho^{2} \nabla \varphi\right)=0 & \text { in } \Omega \\
-\Delta \rho=\frac{1}{\varepsilon^{2}} \rho\left(1-\rho^{2}\right)-\rho|\nabla \varphi|^{2} & \text { in } \Omega \\
\rho=1 & \text { on } \partial \Omega \\
\nabla \varphi \cdot v=0 & \text { on } \partial \Omega
\end{array} .\right.
$$

Thanks to the last equation in (5.9), the global potential $H$ satisfying (5.5) and (5.6) still exists, in this case.

If we consider another non vanishing critical point $v$, then we may write globally $v=u \eta e^{\imath \psi}$, and (5.7) becomes

$$
\left\{\begin{array}{ll}
\operatorname{div}\left(\rho^{2} \eta^{2} \nabla \psi\right)=\operatorname{div}\left(\rho^{2}\left(1-\eta^{2}\right) \nabla \varphi\right) & \text { in } \Omega \\
-\Delta w+a w=\operatorname{Jac}(2 H, w)+\operatorname{div} R+w S+T & \text { in } \Omega \\
\frac{\partial \psi}{\partial v}=0 & \text { on } \partial \Omega \\
w=0 & \text { on } \partial \Omega
\end{array} .\right.
$$

In both cases (i.e., Dirichlet boundary condition or prescribed degrees condition) there is a formula relating $E_{\varepsilon}(v)$ to $E_{\varepsilon}(u)$ :

$$
\begin{aligned}
E_{\varepsilon}(v)= & E_{\varepsilon}(u)+\frac{1}{2} \int_{\Omega}\left[\rho^{2}|\nabla \eta|^{2}+\rho^{2} \eta^{2}|\nabla \psi|^{2}+\left(\eta^{2}-1\right) \operatorname{Jac}(2 H, \psi)\right] \\
& +\frac{1}{2} \int_{\Omega} \frac{1}{2 \varepsilon^{2}} \rho^{4}\left(1-\eta^{2}\right)^{2} .
\end{aligned}
$$

Finally, for further use we discuss the analog of the above formulae when the GL energy is replaced, more generally, by an energy of the form

$$
E(u)=\frac{1}{2} \int_{\Omega}|\nabla u|^{2}+\frac{1}{2} \int_{\Omega} F\left(x,|u|^{2}\right)
$$

here, $F \in C^{1}$. Let $G(x, t)=\frac{\partial F}{\partial t}(x, t)$. Then (5.4) becomes

$$
\begin{cases}\operatorname{div}\left(\rho^{2} \nabla \varphi\right)=0 & \text { in } \Omega \\ -\Delta \rho=-\rho G\left(x, \rho^{2}\right)-\rho|\nabla \varphi|^{2} & \text { in } \Omega \\ \rho=1 & \text { on } \partial \Omega \\ \varphi=\varphi_{0} & \text { on } \partial \Omega\end{cases}
$$

(5.9) is affected similarly. Existence of $H$ still holds, both in the case of Dirichlet and degrees boundary condition.

On the other hand, (5.7) and (5.10) are still valid, with only one change in (5.8): $R, S, T$ are unchanged, while $a$ is given by

$$
a=\rho^{2} \frac{\eta}{\eta-1}\left(G\left(x, \rho^{2} \eta^{2}\right)-G\left(x, \rho^{2}\right)\right) .
$$

Finally, (5.11) becomes

$$
\begin{aligned}
E(v)= & E(u)+\frac{1}{2} \int_{\Omega}\left[\rho^{2}|\nabla \eta|^{2}+\rho^{2} \eta^{2}|\nabla \psi|^{2}+\left(\eta^{2}-1\right) \operatorname{Jac}(2 H, \psi)\right] \\
& +\frac{1}{2} \int_{\Omega}\left[F\left(x, \rho^{2} \eta^{2}\right)-F\left(x, \rho^{2}\right)-\rho^{2}\left(\eta^{2}-1\right) G\left(x, \rho^{2}\right)\right] .
\end{aligned}
$$




\section{Proof of Theorem 1.5}

We consider an energy of the form

$$
E(u)=\frac{1}{2} \int_{\Omega}|\nabla u|^{2}+\frac{1}{2} \int_{\Omega} F\left(x,|u|^{2}\right),
$$

where $F \in C^{1}$ is as in Section 3.

Let $\Omega \subset \mathbb{R}^{2}$ be a $C^{1}$ bounded domain. Denote by $\Gamma_{j}, j \in \llbracket 0, k \rrbracket$, the components of $\partial \Omega$, with $\Gamma_{0}$ enclosing $\Omega$. For prescribed degrees $d_{j}$ on the $\Gamma_{j}$ 's, we let

$$
\mathfrak{M}=\inf \{E(u) ; u \text { has the prescribed degrees }\} .
$$

In the definition of $\mathfrak{M}$ it suffices to consider only maps $u$ such that $|u| \leq 1$. Indeed, we have $E(v) \leq E(u)$, where $v$ minimizes $E$ with boundary condition $u_{\mid \partial \Omega}$. By Lemma 3.2, we have $|v| \leq 1$.

6.1 Lemma. Let $\left(u_{n}\right)$ be a minimizing sequence for $\mathfrak{M}$, satisfying $\left|u_{n}\right| \leq 1$. If (possibly after extracting a subsequence) $u_{n} \rightarrow u$ in $H^{1}(\Omega)$, then

$$
\liminf E\left(u_{n}\right) \geq E(u)+\pi \sum_{j=0}^{k}\left|\operatorname{deg}\left(u_{n}, \Gamma_{j}\right)-\operatorname{deg}\left(u, \Gamma_{j}\right)\right| .
$$

Proof. This result was established in [1, Lemma 1] for the GL energy (without the assumption $\left|u_{n}\right| \leq 1$ ). Under the additional assumption $\left|u_{n}\right| \leq 1$, the proof in [1] applies to general nonlinearities.

6.2 Corollary. Assume that $\mathfrak{M}<\pi$. Then $\mathfrak{M}$ is attained. ${ }^{17}$

Proof. Consider a minimizing sequence $\left(u_{n}\right)$ satisfying $\left|u_{n}\right| \leq 1$. Let, possibly after passing to a subsequence, $u_{n} \rightarrow u$ in $H^{1}(\Omega)$. By Lemma 6.1, we have

$$
\pi>\mathfrak{M} \geq E(u)+\pi \sum_{j=0}^{k}\left|\operatorname{deg}\left(u_{n}, \Gamma_{j}\right)-\operatorname{deg}\left(u, \Gamma_{j}\right)\right|
$$

Thus $u$ has the prescribed degrees and minimal energy.

We next state and prove a generalization of Theorem 1.5.

6.3 Theorem. Let $F \in C^{1}\left(\bar{\Omega} \times \mathbb{R}_{+} ; \mathbb{R}_{+}\right)$satisfy

a) $F(x, 1)=0, \forall x \in \Omega$.

b) $[0,1] \ni t \mapsto F(x, t)$ is convex, $\forall x \in \Omega$.

Then there is some universal ${ }^{18}$ constant $\delta>0$ such that, if $\mathfrak{M}<\delta$, then $\mathfrak{M}$ is attained by a minimizer unique modulo $\mathbb{S}^{1}$.

Before proceeding to the proof of Theorem 6.3 , we note that $F(x, t)=\frac{1}{2 \varepsilon^{2}}(1-t)^{2}$ satisfies the hypotheses of Theorem 6.3, and thus Theorem 6.3 implies Theorem 1.5.

\footnotetext{
${ }^{17}$ The value $\pi$ is sharp: if $\Omega$ is simply connected and we prescribe the degree 1 on the boundary, then $\mathfrak{M}=\pi$ and (at least for GL) $\mathfrak{M}$ is not attained [1, Example 1].

${ }^{18}$ That is, independent of $\Omega$, of the $d_{j}$ 's, and of $F$.
} 
Proof. Assumption a) and b) imply that $[0,1] \ni t \mapsto F(x, t)$ is non increasing. In particular, we may apply Theorem 3.6 to $F$. Let $0<s<1$. Let, with the notations in Theorem 3.6, $\delta=\delta(s)=$ $2 \mathscr{A}_{s, 1}$. If $\mathfrak{M} \leq \delta$, then $\mathfrak{M}$ is attained and, if $u$ minimizes $E$, then $|u| \geq s$ in $\Omega .{ }^{19}$ Let $v$ be another minimizer of $u$. The convexity of $F$ for fixed $x$ implies

$$
F(x, a b)-F(x, b)-b(a-1) \frac{\partial F}{\partial t}(x, b) \geq 0, \quad \forall x \in \Omega, \forall b, a b \in[0,1] .
$$

By combining (6.2) with (5.14), we find that

$$
E(v) \geq E(u)+\frac{1}{2} \int_{\Omega}\left[\rho^{2}|\nabla \eta|^{2}+\rho^{2} \eta^{2}|\nabla \psi|^{2}+\left(\eta^{2}-1\right) \operatorname{Jac}(2 H, \psi)\right] .
$$

Using (6.3), the fact that $s \leq \rho, \rho \eta \leq 1$ and (4.3), we obtain

$$
E(v) \geq E(u)+\frac{s^{2}}{2} \int_{\Omega}|\nabla \eta|^{2}+\frac{s^{2}}{2} \int_{\Omega}|\nabla \psi|^{2}-\frac{2 \sqrt{2}}{s}\|\nabla \eta\|_{L^{2}}\|\nabla H\|_{L^{2}}\|\nabla \psi\|_{L^{2}} .
$$

By combining (5.5) with the identity $|\nabla u|^{2}=\rho^{2}|\nabla \varphi|^{2}+|\nabla \rho|^{2}$, we find that

$$
\|\nabla H\|_{L^{2}}=\left\|\rho^{2} \nabla \varphi\right\|_{L^{2}} \leq\|\rho \nabla \varphi\|_{L^{2}} \leq\|\nabla u\|_{L^{2}} \leq \sqrt{2 \mathfrak{M}} \leq \sqrt{2 \delta},
$$

and thus

$$
E(v) \geq E(u)+\frac{s^{2}}{2} \int_{\Omega}|\nabla \eta|^{2}+\frac{s^{2}}{2} \int_{\Omega}|\nabla \psi|^{2}-\frac{4 \sqrt{\delta}}{s}\|\nabla \eta\|_{L^{2}}\|\nabla \psi\|_{L^{2}} .
$$

Noting that $\delta$ decreases with $s$ and that $\delta(1)=0$, we find that the equation

$$
\frac{4 \sqrt{\delta(s)}}{s}=s^{2}
$$

has exactly one solution. ${ }^{20}$ For this $s$, we find that

$$
E(v) \geq E(u) .
$$

By minimality, we must have $E(v)=E(u)$. If we examine the equality case in (6.7) and use the fact that $\mathfrak{M}<\delta$, we find that equality can occur only if $\nabla \psi=0$ or $\nabla \eta=0$. Going back to (6.4), this implies that we must have both $\nabla \psi=0$ and $\nabla \eta=0$. Thus $v / u$ is constant.

\section{Clearing out}

In the GL jargon, clearing out means absence of vortices, i.e. of zeros. The usual arguments leading to clearing out make use of the global estimate $|\nabla u| \leq \frac{C}{\varepsilon}, 0<\varepsilon<1$, valid if the boundary datum $g$ is smooth and fixed [3]. Such an estimate need not hold if $g$ is merely $H^{1 / 2}$. The result we present below holds for natural boundary datum and for general nonlinearities.

7.1 Proposition. Let $\Omega \subset \mathbb{R}^{2}$ be a $C^{1}$ bounded simply connected domain.

Let $F_{\varepsilon}: \bar{\Omega} \times \mathbb{R}_{+} \rightarrow \mathbb{R}_{+}$satisfy

a) $F_{\varepsilon}(x, 1)=0, \forall x \in \Omega, \forall \varepsilon>0$.

b) $[0,1] \ni t \mapsto F(x, t)$ is non increasing, $\forall x \in \Omega$.

c) $F_{\varepsilon} \in C^{1}(\bar{\Omega} \times[0,1]), \forall \varepsilon>0$.

d) $\lim _{\varepsilon \rightarrow 0} F_{\varepsilon}(x, t)=\infty, \forall x \in \Omega, \forall t \in[0,1)$.

${ }^{19}$ This follows by combining Theorem 3.6 and Example 3.5 a) with Corollary 6.2.

${ }^{20}$ Maple ${ }^{\circledR}$ gives $\delta=0.04518303544 \ldots$ 
Let $g \in H^{1 / 2}\left(\partial \Omega ; \mathbb{S}^{1}\right)$ have zero degree. Let

$$
E_{\varepsilon}(u)=\frac{1}{2} \int_{\Omega}|\nabla u|^{2}+\frac{1}{2} \int_{\Omega} F_{\varepsilon}\left(x,|u|^{2}\right) .
$$

If $u_{\varepsilon}$ minimizes $E_{\varepsilon}$ with boundary datum $g$, then $\left|u_{\varepsilon}\right| \rightarrow 1$ uniformly in $\Omega$ as $\varepsilon \rightarrow 0$.

During the proof, we will use the following elementary fact.

7.2 Lemma. Assume that $f$, g are non negative measurable functions such that

$$
\int_{0}^{\mu} \frac{f(r)}{r} d r<\delta \text { and } \int_{0}^{\mu} r g(r) d r<\delta .
$$

Then there is some $r \in(0, \mu)$ such that

$$
f(r)<4 \delta \text { and } g(r)<4 \frac{\delta}{\mu^{2}} .
$$

Proof of Lemma 7.2. We have

$$
\int_{0}^{\mu}\left(\frac{f(r)}{r}+r g(r)\right) d r<2 \delta=\int_{0}^{\mu} \frac{4 \delta r}{\mu^{2}} d r .
$$

From the mean value theorem, there exists some $r$ such that $\frac{f(r)}{r}+r g(r)<\frac{4 \delta r}{\mu^{2}}$. It is easy to see that this $r$ has all the required properties.

Proof of Proposition 7.1. In the first part of the proof, we establish the $H^{1}$-convergence of the family $\left(u_{\varepsilon}\right)$. We follow essentially [3, Proposition 1]. Since $\operatorname{deg}(g, \partial \Omega)=0$, we may write $g=e^{\imath \varphi_{0}}$, with $\varphi_{0} \in H^{1 / 2}(\partial \Omega ; \mathbb{R})$ (Section $\left.5 \mathrm{c}\right)$ ). Let $\Phi$ be the harmonic extension of $\varphi_{0}$ and set $u_{0}=e^{\imath \Phi}$. Then $u_{0}$ is the unique minimizer of

$$
\left\{u \in H^{1}\left(\Omega ; \mathbb{S}^{1}\right) ; u_{\mid \partial \Omega}=g\right\} \ni u \mapsto \frac{1}{2} \int_{\Omega}|\nabla u|^{2}
$$

(Section $5 \mathrm{~m})$ ).

Since $E_{\varepsilon}\left(u_{\varepsilon}\right) \leq E_{\varepsilon}\left(u_{0}\right)=C_{0}$, we find that $\int_{\Omega} F_{\varepsilon}\left(x,\left|u_{\varepsilon}\right|^{2}\right) \leq 2 C_{0}$. This inequality combined with the fact that $\left|u_{\varepsilon}\right| \leq 1$ (by Lemma 3.2) and with property d) of $F_{\varepsilon}$ implies that $\left|u_{\varepsilon}\right| \rightarrow 1$ a.e. As in the proof of [3, Proposition 1], this implies that $u_{\varepsilon} \rightarrow u_{0}$ in $H^{1}(\Omega)$. As a byproduct, we obtain

$$
\lim _{\varepsilon \rightarrow 0} \int_{\Omega} F_{\varepsilon}\left(x,\left|u_{\varepsilon}\right|^{2}\right)=0 .
$$

Next, we prove that $\left|u_{\varepsilon}\right| \rightarrow 1$ uniformly possibly except a small boundary layer. This part of the proof is partly inspired by the approach in [24].

Let $\bar{d}: \bar{\Omega} \rightarrow \mathbb{R}_{+}$be a regularized distance to $\partial \Omega$. More specifically, we assume that $\bar{d} \in C^{\infty}(\Omega)$, and

$$
\frac{1}{2} \operatorname{dist}(x, \partial \Omega) \leq \bar{d}(x) \leq \operatorname{dist}(x, \partial \Omega), \quad \forall x \in \Omega .
$$

Let $\delta>0$ to be fixed later. Since $u_{\varepsilon} \rightarrow u_{0}$ in $H^{1}(\Omega)$, there are $\mu>0$ and $\varepsilon_{0}>0$ such that

$$
\int_{\mathbb{D}(x, \mu)}\left|\nabla u_{\varepsilon}\right|^{2}<\delta, \quad \forall x \in \Omega, \forall \varepsilon<\varepsilon_{0}
$$

and

$$
\int_{\{x \in \Omega ; \operatorname{dist}(x, \partial \Omega)<\mu\}}\left|\nabla u_{\varepsilon}\right|^{2}<\delta, \quad \forall \varepsilon<\varepsilon_{0} .
$$


In addition, we may also assume that

$$
\int_{\Omega} F_{\varepsilon}\left(x,\left|u_{\varepsilon}\right|^{2}\right)<\delta, \quad \forall \varepsilon<\varepsilon_{0} .
$$

By Sard's lemma, we may also assume that $\mu$ has the following property: the set

$$
\omega_{\mu}=\{x \in \Omega ; \bar{d}(x)<\mu\}
$$

is smooth, with boundary $\partial \Omega \cup \Gamma_{\mu}$, where

$$
\Gamma_{\mu}=\{x \in \Omega ; \bar{d}(x)=\mu\} .
$$

Let $x \in \Omega \backslash \omega_{\mu}$. For $\varepsilon<\varepsilon_{0}$, (7.1) and (7.3) combined with Lemma 7.2 applied with

$$
f(r)=r \int_{C(x, r)}\left|\nabla u_{\varepsilon}\right|^{2}, g(r)=\frac{1}{r} \int_{C(x, r)} F_{\varepsilon}\left(y,\left|u_{\varepsilon}\right|^{2}\right)
$$

imply the existence of an $r \in(0, \mu)$ such that

$$
\frac{1}{2 \pi}\left(\int_{C(x, r)}\left|\nabla u_{\varepsilon}\right|\right)^{2} \leq r \int_{C(x, r)}\left|\nabla u_{\varepsilon}\right|^{2}<4 \delta
$$

and

$$
2 \pi \min _{C(x, r)} F_{\varepsilon}\left(y,\left|u_{\varepsilon}\right|^{2}\right) \leq \frac{1}{r} \int_{C(x, r)} F_{\varepsilon}\left(x,\left|u_{\varepsilon}\right|^{2}\right)<4 \delta .
$$

We fix now some $t \in(0,1)$. If $\varepsilon_{0}$ and $\frac{\delta}{\mu}$ are sufficiently small, ${ }^{21}$ then (7.6) combined with properties b) and d) of $F_{\varepsilon}$ implies that for some $y \in C(x, r)$ we have (possibly after rotating $u_{\varepsilon}$ )

$$
u_{\varepsilon}(y) \in \mathbb{R} \text { and } u_{\varepsilon}(y)>\frac{2+t}{3} .
$$

On the other hand, (7.5) implies that

$$
\left|u_{\varepsilon}(y)-u_{\varepsilon}(z)\right| \leq \sqrt{8 \pi \delta \mu^{2}}, \quad \forall z \in C(x, r) .
$$

This fact combined with (7.7) implies that

$$
u_{\varepsilon}(C(x, r)) \subset\left\{z \in \mathbb{C} ; \operatorname{Re} \mathrm{z} \geq \frac{1+t}{2}\right\},
$$

provided $\varepsilon_{0}, \delta$ and $\frac{\delta}{\mu}$ are sufficiently small. We are now in position to invoke Theorem 3.6 to infer that

$$
u_{\varepsilon}(\mathbb{D}(x, r)) \subset\left\{z \in \mathbb{C} ; \operatorname{Re} \mathrm{z} \geq \frac{1+t}{2}\right\} .
$$

In particular, we have

$$
\left|u_{\varepsilon}(x)\right| \geq \frac{1+t}{2} \quad \forall x \in \Omega \backslash \omega_{\mu}, \forall \varepsilon<\varepsilon_{0} .
$$

We next rule out the case of the boundary layer. In view of (7.4) and (7.8), we have

$$
\left|u_{\varepsilon}\right| \geq \frac{1+t}{2} \text { on } \partial \omega_{\mu}, \quad \forall \varepsilon<\varepsilon_{0} .
$$

By combining (7.9) with Example 3.5 c), (7.2) and with Theorem 3.6, we find that $\left|u_{\varepsilon}\right| \geq t$ in $\omega_{\mu}$, provided $\delta$ is sufficiently small.

Finally, for $\varepsilon<\varepsilon_{0}$ we have $\left|u_{\varepsilon}\right| \geq t$ in $\Omega$. The proof of Proposition 7.1 is complete.

\footnotetext{
${ }^{21}$ Smallness depending on $t$, not on $x$ or $\varepsilon$.
} 


\section{Proof of Theorem 1.2}

Throughout this section, $\Omega \subset \mathbb{R}^{2}$ is a $C^{1}$ bounded simply connected domain.

We start with a slight generalization of Theorem $1.2 \mathrm{~b}$ ).

8.1 Theorem. Let $F \in C^{1}\left(\bar{\Omega} \times \mathbb{R}_{+} ; \mathbb{R}_{+}\right)$satisfy

a) $F(x, 1)=0, \forall x \in \Omega$.

b) $[0,1] \ni t \mapsto F(x, t)$ is convex, $\forall x \in \Omega$.

Let

$$
E(u)=\frac{1}{2} \int_{\Omega}|\nabla u|^{2}+\frac{1}{2} \int_{\Omega} F\left(x,|u|^{2}\right) .
$$

Let $g \in H^{1 / 2}\left(\partial \Omega ; \mathbb{S}^{1}\right)$ have zero degree.

There is some universal constant $\delta>0$ such that, if $|g|_{H^{1 / 2}(\partial \Omega)}<\delta$, then $E$ has a unique minimizer with Dirichlet datum $g$.

Proof. By Section $5 \mathrm{~h})$, for small $\delta$ there is some $v \in H^{1}\left(\Omega ; \mathbb{S}^{1}\right)$ such that $v_{\mid \partial \Omega}=g$ and $\int_{\Omega}|\nabla v|^{2}<$ $C^{2} \delta^{2}$. In particular, if

$$
\mathfrak{M}=\min \{E(u) ; u=g \text { on } \partial \Omega\},
$$

then $\mathfrak{M} \leq E(v)<C^{2} \delta^{2}$. Let $0<s<1$. By Theorem 3.6 and Remark 3.4 a), we have $|u| \geq s$ in $\Omega$, provided $\delta$ is sufficiently small. We may now argue as in the proof of Theorem 6.3.

We continue with a more general form of Theorem $1.2 \mathrm{a}$ ).

8.2 Theorem. Let $F_{\varepsilon} \in C^{1}\left(\bar{\Omega} \times \mathbb{R}_{+} ; \mathbb{R}_{+}\right)$satisfy

a) $F_{\varepsilon}(x, 1)=0, \forall x \in \Omega$.

b) $[0,1] \ni t \mapsto F_{\varepsilon}(x, t)$ is convex, $\forall x \in \Omega, \forall \varepsilon>0$.

c) $\lim _{\varepsilon \rightarrow 0} \underset{t \in[s, 1]}{\operatorname{essinf}} \frac{\partial^{2} F_{\varepsilon}}{\partial t^{2}}(x, t)=\infty, \forall x \in \Omega, \forall s \in[0,1)$.

Let

$$
E_{\varepsilon}(u)=\frac{1}{2} \int_{\Omega}|\nabla u|^{2}+\frac{1}{2} \int_{\Omega} F_{\varepsilon}\left(x,|u|^{2}\right) .
$$

Let $g \in H^{1 / 2}\left(\partial \Omega ; \mathbb{S}^{1}\right)$ have zero degree. Then, for small $\varepsilon, E_{\varepsilon}$ has a unique minimizer with Dirichlet datum $g$.

Proof. Let us start by noting that properties a)-c) of $F_{\varepsilon}$ imply

d) $\lim _{\varepsilon \rightarrow 0} F_{\varepsilon}(x, t)=\infty, \forall x \in \Omega, \forall t \in[0,1)$.

e) $\lim _{\varepsilon \rightarrow 0} \inf _{\rho, \rho \eta \in[s, 1]} \rho^{2} \frac{\eta}{\eta-1}\left(\frac{\partial F_{\varepsilon}}{\partial t}\left(x, \rho^{2} \eta^{2}\right)-\frac{\partial F_{\varepsilon}}{\partial t}\left(x, \rho^{2}\right)\right)=\infty, \forall x \in \Omega, \forall s \in[0,1)$.

Write $g=e^{\imath \varphi_{0}}$, with $\varphi_{0} \in H^{1 / 2}(\partial \Omega ; \mathbb{R})(c f$ Section $5 \mathrm{c})$ ). Let $\Phi$ be the harmonic extension of $\varphi_{0}$. Let $u=u_{\varepsilon}, v=v_{\varepsilon}$ be minimizers of $E_{\varepsilon}$.

By Proposition 7.1, we have

$$
|u| \rightarrow 1 \quad \text { uniformly in } \Omega \text { as } \varepsilon \rightarrow 0 .
$$

By Section $5 \mathrm{j}$ ), we may write, for small $\varepsilon, u=\rho e^{\imath \varphi}$, with $\rho=\rho_{\varepsilon}=|u| \in H^{1}(\Omega)$ and $\varphi=\varphi_{\varepsilon} \in H^{1}(\Omega)$ satisfy $\rho \rightarrow 1$ uniformly in $\Omega$ as $\varepsilon \rightarrow 0$ and $\varphi_{\mid \partial \Omega}=\varphi_{0}$. We claim that

$$
\varphi \rightarrow \Phi \quad \text { in } H^{1}(\Omega) \text { as } \varepsilon \rightarrow 0 .
$$


Indeed, this follows from (8.1) combined with the equation

$$
\begin{cases}\operatorname{div}\left(\rho^{2} \nabla(\varphi-\Phi)\right)=\operatorname{div}\left(\left(1-\rho^{2}\right) \nabla \Phi\right) & \text { in } \Omega \\ \varphi-\Phi=0 & \text { on } \partial \Omega\end{cases}
$$

satisfied by $\varphi-\Phi, c f(5.4)$.

Let $H=H_{\varepsilon}$ be defined by (5.5). By (8.2), we have

$$
H \rightarrow H_{0} \quad \text { in } H^{1}(\Omega),
$$

where $H_{0}$ is defined by

$$
\frac{\partial H_{0}}{\partial x}=\frac{\partial \Phi}{\partial y}, \frac{\partial H_{0}}{\partial y}=-\frac{\partial \Phi}{\partial x}, \quad \int_{\Omega} H_{0}=0 .
$$

Write, for small $\varepsilon, v=u \eta e^{\imath \psi}$, with $\eta=\eta_{\varepsilon}, \psi=\psi_{\varepsilon}$ and $w=w_{\varepsilon}=1-\eta_{\varepsilon}$ satisfying

$$
w \rightarrow 0 \quad \text { uniformly in } \Omega \text { as } \varepsilon \rightarrow 0
$$

and (5.10). Property e) of $F_{\varepsilon}$ implies that $a=a_{\varepsilon}$ given by (5.13) satisfies

$$
a(x) \rightarrow \infty \quad \text { as } \varepsilon \rightarrow 0, \forall x \in \Omega .
$$

Thanks to (5.8), (5.10), (8.3), (8.4) and (8.5), we are in position to apply Lemma 4.4 in order to obtain

$$
\|\nabla \eta\|_{L^{2}}=\|\nabla w\|_{L^{2}}=o\left(\|\nabla \psi\|_{L^{2}}\right) \quad \text { as } \varepsilon \rightarrow 0 .
$$

As noted in the proof of Theorem 6.3, property b) of $F_{\varepsilon}$ used in conjunction with (5.14) implies that

$$
E_{\varepsilon}(v) \geq E_{\varepsilon}(u)+\frac{1}{2} \int_{\Omega}\left[\rho^{2}|\nabla \eta|^{2}+\rho^{2} \eta^{2}|\nabla \psi|^{2}+\left(\eta^{2}-1\right) \operatorname{Jac}(2 H, \psi)\right] .
$$

In turn, (8.7) combined with (4.3) implies that, for a fixed $s \in(0,1)$ and sufficiently small $\varepsilon$,

$$
E_{\varepsilon}(v) \geq E_{\varepsilon}(u)+\frac{s^{2}}{2} \int_{\Omega}|\nabla \eta|^{2}+\frac{s^{2}}{2} \int_{\Omega}|\nabla \psi|^{2}-\frac{2 \sqrt{2}}{s}\|\nabla \eta\|_{L^{2}}\|\nabla H\|_{L^{2}}\|\nabla \psi\|_{L^{2}} .
$$

By combining (8.3), (8.6) and (8.8) we find that, for small $\varepsilon$, we have

$$
E_{\varepsilon}(v) \geq E_{\varepsilon}(u)+\frac{s^{2}}{2} \int_{\Omega}|\nabla \eta|^{2} .
$$

The minimality of $u$ and $v$ combined with (8.9) implies that $\eta=1$, and thus, going back to (8.7), that $u=v$.

\section{More}

\subsection{GL critical points with Dirichlet boundary condition}

Our result here is

9.1 Theorem. Let $\Omega \subset \mathbb{R}^{2}$ be a $C^{1}$ bounded simply connected domain. Let $g \in H^{1 / 2}\left(\partial \Omega ; \mathbb{S}^{1}\right)$ have zero degree. Let $u=u_{\varepsilon}$ be a critical point of the GL energy with datum $g$. Assume that:

Either

$$
\text { Or }
$$

a) $|u| \geq c$, with $c \in(0,1)$ independent of small $\varepsilon$.

b) $E_{\varepsilon}(u) \leq C$, with $C>0$ independent of small $\varepsilon$. 
Then, for sufficiently small $\varepsilon, u$ is the minimizer of $E_{\varepsilon}$ with datum $g$.

When $g$ is smooth, Theorem 9.1 was proved in [12]. The approach there relies on global pointwise estimates for $\nabla u$ and does not apply to our case.

Proof of Theorem 9.1. The plan is to prove the following:

$$
a) \Longrightarrow b) \Longrightarrow|u| \rightarrow 1 \text { uniformly in } \Omega \text { as } \varepsilon \rightarrow 0 .
$$

Indeed, assume (9.1) proved, for the moment. In other words, we have established the validity of (8.1) for a critical point satisfying a) or b). Since (8.1) also holds (by Proposition 7.1) for a minimizer $v=v_{\varepsilon}$ of $E_{\varepsilon}$ with datum $g$, we are in position to repeat the proof of Theorem 8.2 and conclude that, for small $\varepsilon$, we have $u=v$. It thus remains to prove (9.1).

Step 1 in the proof of (9.1): $a) \Longrightarrow b$ ). As in Section 5 c), write $g=e^{\imath \varphi_{0}}$, with $\varphi_{0} \in H^{1 / 2}(\partial \Omega$; $\mathbb{R})$. By Section $5 \mathrm{k}$ ), we may write $u=\rho e^{\imath \varphi}$, with $\rho=\rho_{\varepsilon}=|u|, c \leq \rho \leq 1$, while $\varphi=\varphi_{\varepsilon} \in H^{1}(\Omega)$ satisfies $\varphi_{\mid \partial \Omega}=\varphi_{0}$. Then

$$
E_{\varepsilon}(u)=\frac{1}{2} \int_{\Omega} \rho^{2}|\nabla \varphi|^{2}+\frac{1}{2} \int_{\Omega}|\nabla \rho|^{2}+\frac{1}{4 \varepsilon^{2}} \int_{\Omega}\left(1-\rho^{2}\right)^{2} .
$$

Using (5.4) and the fact that $c \leq \rho \leq 1$, we find that

$$
\|\nabla \varphi\|_{L^{2}} \leq C
$$

in particular, the first integral in (9.2) is uniformly bounded. (9.3) also implies that $H=H_{\varepsilon}$ given by (5.5) satisfies

$$
\|\nabla H\|_{L^{2}} \leq C .
$$

Let $\zeta=\zeta_{\varepsilon}=1-\rho$. We rewrite the second equation in (5.4) in the equivalent form

$$
\left\{\begin{array}{ll}
-\Delta \zeta+\frac{\rho(1+\rho)}{\varepsilon^{2}} \zeta=\frac{1}{1-\zeta} \operatorname{Jac}(H, \varphi) & \text { in } \Omega \\
\zeta=0 & \text { on } \partial \Omega
\end{array} .\right.
$$

If we multiply (9.5) by $\zeta$ and use (4.3) combined with (9.3) and (9.4) and with the inequality

$$
\left\|\nabla\left(\frac{\zeta}{1-\zeta}\right)\right\|_{L^{2}} \leq C\|\nabla \zeta\|_{L^{2}}=C\|\nabla \rho\|_{L^{2}}
$$

we find that

$$
\int_{\Omega}|\nabla \rho|^{2}+\frac{1}{\varepsilon^{2}} \int_{\Omega}\left(1-\rho^{2}\right)^{2} \leq \int_{\Omega}|\nabla \zeta|^{2}+C \int_{\Omega} \frac{\rho(1+\rho)}{\varepsilon^{2}} \zeta^{2} \leq C\|\nabla \rho\|_{L^{2}}
$$

Inequality (9.6) implies that the second and the third integral in (9.2) are uniformly bounded, and thus b) holds.

Step 2 in the proof of (9.1): $b) \Longrightarrow|u| \rightarrow 1$ uniformly in $\Omega$ as $\varepsilon \rightarrow 0$. We rely on the following

9.2 Lemma ([19]). Let $C>0$ and $t \in(0,1)$. There exists some $\varepsilon_{0}=\varepsilon_{0}(C, t)$ such that, if $\varepsilon<r \varepsilon_{0}$ and $u: \mathbb{D}(z, r) \rightarrow \mathbb{C}$ is a critical point of $G L$ in $\mathbb{D}(z, r)$ satisfying $|u| \leq 1$ and $E_{\varepsilon}(u) \leq C$, then $|u(z)| \geq t$.

9.3 Lemma. We have

$$
|\nabla u(x)| \leq C \max \left\{\frac{1}{\varepsilon}, \frac{1}{\operatorname{dist}(x, \partial \Omega)}\right\}, \quad \forall x \in \Omega, \forall \varepsilon>0 .
$$


Proof of Lemma 9.3. Let $u_{0}$ be the harmonic extension of $g$ and set $v=u-u_{0}$. By Lemma 3.2, we have $|u| \leq 1$, and thus $v$ satisfies

$$
\left\{\begin{array}{ll}
|\Delta v| \leq \frac{1}{\varepsilon^{2}} & \text { in } \Omega \\
v=0 & \text { on } \partial \Omega
\end{array} .\right.
$$

We obtain (9.7) by combining the straightforward estimate

$$
\left|\nabla u_{0}(x)\right| \leq \frac{C}{\operatorname{dist}(x, \partial \Omega)}, \quad \forall x \in \Omega,
$$

with the following Gagliardo-Nirenberg type estimate valid for solutions of (9.8) [3]

$$
|\nabla v(x)| \leq C \max \left\{1, \frac{1}{\varepsilon}\right\}, \quad \forall x \in \Omega, \forall \varepsilon>0 .
$$

Proof of Step 2 continued. Let $t \in(0,1)$ be fixed. With $C$ as in b), let $\varepsilon_{0}$ be given by Lemma 9.2.

Let $x \in \Omega$ be such that $\operatorname{dist}(x, \partial \Omega)>\varepsilon / \varepsilon_{0}$. By Lemma 9.2 , we have $|u(x)| \geq t$. We complete Step 2 if we prove that, for small $\varepsilon$ and for $x \in \Omega$ such that $\operatorname{dist}(x, \partial \Omega) \leq \varepsilon / \varepsilon_{0}$, we have $|u(x)| \geq t$. Argue by contradiction and assume that there are sequences $\varepsilon_{n} \rightarrow 0$ and $x_{n}$ such that:

$$
\left|u_{\varepsilon_{n}}\left(x_{n}\right)\right|<t, \operatorname{dist}\left(x_{n}, \partial \Omega\right)=\lambda_{n} \frac{\varepsilon_{n}}{\varepsilon_{0}}, \lambda_{n} \rightarrow \lambda \in[0,1] .
$$

We rule out (9.9) via a blow up analysis. We let $y_{n}$ be a projection of $x_{n}$ onto $\partial \Omega$. After rotation and translation, we may assume that $y_{n}=0$ and that $x_{n}=\imath \lambda_{n} \frac{\varepsilon_{n}}{\varepsilon_{0}}$. Set $v_{n}(z)=u_{\varepsilon_{n}}\left(\lambda_{n} \frac{\varepsilon_{n}}{\varepsilon_{0}} z\right)$. Let $\mu=\frac{\lambda^{2}}{\varepsilon_{0}^{2}}$. By (9.7), b) and the fact that the boundary datum of $u_{\varepsilon_{n}}$ is fixed (and thus the blow up of the boundary datum is constant), we find that $\left(v_{n}\right)$ converges, possibly up to a subsequence, uniformly on compacts of

$$
U=\{z \in \mathbb{C} ; \operatorname{Im} z>0\}
$$

to a map $v: U \rightarrow \mathbb{C}$ satisfying

$$
\begin{aligned}
& -\Delta v=\mu v\left(1-|v|^{2}\right) \quad \text { in } U, \\
& \int_{U}|\nabla v|^{2}+\mu \int_{U}\left(1-|v|^{2}\right)^{2}<\infty \\
& |v(l)| \leq t<1
\end{aligned}
$$

and

$$
v_{\mid \mathbb{R}}=\alpha \in \mathbb{S}^{1} .
$$

We complete the proof of Step 2, and thus of Theorem 9.1, with the help the next result.

9.4 Lemma. Let $v$ satisfy (9.10), (9.11) and (9.13). Then $v=\alpha$. 
Proof of Lemma 9.4. By (9.11), there is a sequence $R_{n} \rightarrow \infty$ such that

$$
\lim _{n \rightarrow \infty} R_{n} \int_{C_{R_{n}} \cap U}\left[|\nabla v|^{2}+\mu\left(1-|v|^{2}\right)^{2}\right]=0 .
$$

Consider first the case where $\mu>0$. Pohozaev's identity yields

$$
\begin{aligned}
\frac{\mu}{2} \int_{\mathbb{D}_{R_{n}} \cap U}\left(1-|v|^{2}\right)^{2}+\frac{R_{n}}{2} \int_{C_{R_{n}} \cap U}\left|\frac{\partial v}{\partial r}\right|^{2}= & \frac{\mu R_{n}}{4} \int_{C_{R_{n}} \cap U}\left(1-|v|^{2}\right)^{2} \\
& +\frac{R_{n}}{2} \int_{C_{R_{n}} \cap U}\left|\frac{\partial v}{\partial \tau}\right|^{2}
\end{aligned}
$$

By letting $n \rightarrow \infty$, we find that $|v|=1$. In turn, this implies (using (9.10)),

$$
0=\Delta|v|^{2}=2|\nabla v|^{2}
$$

so that $v=\alpha$.

Assume next that $\mu=0$. Set $w=v-\alpha$. Using the fact that $w(R)=0, w(-R)=0$, the scaled version of Poincaré's inequality gives

$$
\int_{C_{R} \cap U}|w|^{2} \leq C R^{2} \int_{C_{R} \cap U}|\nabla w|^{2}
$$

Thus

$$
\int_{\mathbb{D}_{R_{n}} \cap U}|\nabla w|^{2}=\int_{C_{R_{n}} \cap U} w \cdot \frac{\partial w}{\partial r} \leq C R_{n} \int_{C_{R_{n}} \cap U}|\nabla w|^{2}=C R_{n} \int_{C_{R_{n}} \cap U}|\nabla v|^{2} .
$$

By letting $n \rightarrow \infty$, we find that $w$ is constant, and thus $v=\alpha$.

\subsection{GL critical points with prescribed degrees}

Our main result in this section concerns uniqueness of GL critical points with prescribes degrees and small energy. Unlike in Theorem 1.5, the notion of smallness we consider depends on the geometric properties of the domain.

More specifically, let $s>1$. For $x \in \Omega$, we consider the following set:

$$
A_{x}=\left\{\theta \in[0,2 \pi] ; \text { the segment }\left\{x+t e^{\imath \theta} ; \operatorname{dist}(x, \partial \Omega) \leq t \leq s \operatorname{dist}(x, \partial \Omega)\right\} \operatorname{intersects} \mathbb{R}^{2} \backslash \Omega\right\} .
$$

Let $a \in(0, \pi)$. We say that $\Omega \subset \mathbb{R}^{2}$ has the property $\left(P_{s, a}\right)$ if the measure of $A_{x}$ is at least $a$, for each $x \in \Omega{ }^{22}$ Intuitively, this property measures at the same time the flatness of $\partial \Omega$ and the width of $\Omega$ : if $\Omega$ has the property $\left(P_{s, a}\right)$ for $s$ close to 1 and $a$ close to $\pi$, then $\partial \Omega$ is almost flat and $\Omega$ is thin. In particular, let us note that, given $s>1$ and $a \in(0, \pi)$, the circular annulus $\mathbb{D}_{\mathbb{R}} \backslash \mathbb{D}$ satisfies $\left(P_{s, a}\right)$ provided $R$ is sufficiently close to 1 . Therefore, our next result generalizes, to critical points, Theorem 1.4 obtained in [14] for minimizers.

9.5 Theorem. Let $\Omega$ be a $C^{1}$ bounded domain. Let $u$ be a critical point of $E_{\varepsilon}$ with prescribed degrees $d_{j}, j \in \llbracket 0, k \rrbracket$, on the components $\Gamma_{j}, j \in \llbracket 0, k \rrbracket$, of $\partial \Omega$. There is some $\delta=\delta(s, a)$ such that, if $E_{\varepsilon}(u)<\delta$, then $u$ is the unique (modulo $2 \pi$ ) minimizer of $E_{\varepsilon}$ with these prescribed degrees.

Proof. If we take a look at the proof of Theorem 6.3, we see that it suffices to prove that $|u| \geq \frac{1}{2}$ in $\Omega$, provided $\delta$ is sufficiently small.

We note that $|u| \leq 1$, by the maximum principle [3]. ${ }^{23}$ Let $\delta \leq 1$ to be fixed later. By Lemma 9.2 , there is some $\varepsilon$ (independent of $\delta \leq 1$ ) such that $|u(x)| \geq \frac{1}{2} \operatorname{provided} \operatorname{dist}(x, \partial \Omega) \geq \frac{\varepsilon}{\varepsilon_{0}}$. It

${ }^{22}$ This definition is inspired by the proof of the main result in [14]. So is the proof of Theorem 9.5.

${ }^{23}$ There, $u_{\mid \partial \Omega}$ is supposed smooth, but the proof applies to a general boundary datum. 
remains to prove that $|u(x)| \geq \frac{1}{2}$ provided $\operatorname{dist}(x, \partial \Omega)<\frac{\varepsilon}{\varepsilon_{0}}$. This is obtained by contradiction: assume the existence of an $x$ satisfying

$$
|u(x)|<\frac{1}{2} \text { and } \operatorname{dist}(x, \partial \Omega)<\frac{\varepsilon}{\varepsilon_{0}} .
$$

By Lemma 9.3, there is some $C \in(0,1 / 2)$ (again, independent of $\delta \leq 1$ ) such that $|u(y)| \leq \frac{3}{4}$ provided $|x-y| \leq C \operatorname{dist}(x, \partial \Omega)$. In particular, we have

$$
\left|u\left(x+C \operatorname{dist}(x, \partial \Omega) e^{\imath \theta}\right)\right| \leq \frac{3}{4}, \quad \forall \theta \in[0,2 \pi] .
$$

Let now $\theta \in A_{x}$. For such $\theta$, we have $\left|u\left(x+t e^{\imath \theta}\right)\right|=1$ for some

$$
t=t(\theta) \in[\operatorname{dist}(x, \partial \Omega), s \operatorname{dist}(x, \partial \Omega)]
$$

such that $\left(x, x+t e^{\imath \theta}\right) \subset \Omega$. We find that

$$
\int_{C \operatorname{dist}(x, \partial \Omega)}^{t}\left|\nabla u\left(x+r e^{\imath \theta}\right)\right| d r \geq \frac{1}{4} .
$$

Consequently, we have

$$
\int_{C \operatorname{dist}(x, \partial \Omega)}^{t} r\left|\nabla u\left(x+r e^{\imath \theta}\right)\right|^{2} d r \geq \frac{1}{16 \ln (t / C \operatorname{dist}(x, \partial \Omega))} \geq K,
$$

for some constant $K>0$ independent of $x$ and $\theta \in A_{x}$.

If we integrate (9.17) with respect to $\theta \in A_{x}$, we find that

$$
2 \delta \geq 2 E_{\varepsilon}(u) \geq \int_{\Omega}|\nabla u|^{2} \geq \int_{A_{x}}\left(\int_{C \operatorname{dist}(x, \partial \Omega)}^{t} r\left|\nabla u\left(x+r e^{\imath \theta}\right)\right|^{2} d r\right) d \theta \geq a K .
$$

This is impossible for small $\delta$. This contradiction completes the proof of Theorem 9.5.

\section{References}

[1] L. Berlyand and P. Mironescu. Ginzburg-Landau minimizers with prescribed degrees. Capacity of the domain and emergence of vortices. J. Funct. Anal., 239(1):76-99, 2006.

[2] L. Berlyand and P. Mironescu. Two-parameter homogenization for a Ginzburg-Landau problem in a perforated domain. Netw. Heterog. Media, 3(3):461-487, 2008.

[3] F. Bethuel, H. Brezis, and F. Hélein. Asymptotics for the minimization of a GinzburgLandau functional. Calc. Var. Partial Differential Equations, 1(2):123-148, 1993.

[4] F. Bethuel, H. Brezis, and F. Hélein. Ginzburg-Landau vortices. Progress in Nonlinear Differential Equations and their Applications, 13. Birkhäuser Boston Inc., Boston, MA, 1994.

[5] F. Bethuel, H. Brezis, and G. Orlandi. Small energy solutions to the Ginzburg-Landau equation. C. R. Acad. Sci. Paris Sér. I Math., 331(10):763-770, 2000.

[6] F. Bethuel and J.-M. Ghidaglia. Improved regularity of solutions to elliptic equations involving Jacobians and applications. J. Math. Pures Appl. (9), 72(5):441-474, 1993.

[7] J. Bourgain, H. Brezis, and P. Mironescu. Lifting in Sobolev spaces. J. Anal. Math., $80: 37-86,2000$.

[8] H. Brezis. Degree theory: old and new. In Topological nonlinear analysis, II (Frascati, 1995), volume 27 of Progr. Nonlinear Differential Equations Appl., pages 87-108. Birkhäuser Boston, Boston, MA, 1997. 
[9] H. Brezis and P. Mironescu. Sobolev Maps with Values into the Circle. Analytical, Geometrical and Topological Aspects. in preparation.

[10] H. Brezis and L. Nirenberg. Degree theory and BMO. I. Compact manifolds without boundaries. Selecta Math. (N.S.), 1(2):197-263, 1995.

[11] H. Brezis and L. Nirenberg. Degree Theory and BMO, Part II: Compact manifolds with boundaries. Selecta Math. (N.S.), 2:309-368, 1996.

[12] M. Comte and P. Mironescu. Minimizing properties of arbitrary solutions to the GinzburgLandau equation. Proc. Roy. Soc. Edinburgh Sect. A, 129(6):1157-1169, 1999.

[13] M. Dos Santos, P. Mironescu, and O. Misiats. The Ginzburg-Landau functional with a discontinuous and rapidly oscillating pinning term. Part I: the zero degree case. Commun. Contemp. Math., 13(5):1-30, 2011.

[14] D. Golovaty and L. Berlyand. On uniqueness of vector-valued minimizers of the Ginzburg-Landau functional in annular domains. Calc. Var. Partial Differential Equations, 14(2):213-232, 2002.

[15] M.A. Krasnosel'ski1. Positive solutions of operator equations. Translated from the Russian by Richard E. Flaherty; edited by Leo F. Boron. P. Noordhoff Ltd. Groningen, 1964.

[16] L. Lassoued and P. Mironescu. Ginzburg-Landau type energy with discontinuous constraint. J. Anal. Math., 77:1-26, 1999.

[17] F.H. Lin and T. Rivière. Complex Ginzburg-Landau equations in high dimensions and codimension two area minimizing currents. J. Eur. Math. Soc. (JEMS), 1(3):237-311, 1999.

[18] F.H. Lin and T. Rivière. A quantization property for static Ginzburg-Landau vortices. Comm. Pure Appl. Math., 54(2):206-228, 2001.

[19] P. Mironescu. Explicit bounds for solutions to a Ginzburg-Landau type equation. Rev. Roumaine Math. Pures Appl., 41(3-4):263-271, 1996.

[20] P. Mironescu. Les minimiseurs locaux pour l'équation de Ginzburg-Landau sont à symétrie radiale. C. R. Acad. Sci. Paris Sér. I Math., 323(6):593-598, 1996.

[21] F. Murat. Compacité par compensation. Ann. Scuola Norm. Sup. Pisa Cl. Sci. (4), 5(3):489-507, 1978.

[22] F. Pacard and T. Rivière. Linear and nonlinear aspects of vortices. Progress in Nonlinear Differential Equations and their Applications, 39. Birkhäuser Boston Inc., Boston, MA, 2000. The Ginzburg-Landau model.

[23] T. Rivière. Line vortices in the U(1)-Higgs model. ESAIM Contrôle Optim. Calc. Var., 1:77-167 (electronic), 1995/96.

[24] M. Struwe. On the asymptotic behavior of minimizers of the Ginzburg-Landau model in 2 dimensions. Differential Integral Equations, 7(5-6):1613-1624, 1994.

[25] H.C. Wente. An existence theorem for surfaces of constant mean curvature. J. Math. Anal. Appl., 26:318-344, 1969.

[26] D. Ye and F. Zhou. Uniqueness of solutions of the Ginzburg-Landau problem. Nonlinear Anal., 26(3):603-612, 1996. 